\title{
THE VARIETY GENERATED BY ORDER ALGEBRAS
}

\author{
R. FREESE, J. JEŽEK, P. JIPSEN, \\ P. MARKOVIĆ, M. MARÓTI, R. MCKENZIE
}

\begin{abstract}
Every ordered set can be considered as an algebra in a natural way. We investigate the variety generated by order algebras. We prove, among other things, that this variety is not finitely based and, although locally finite, it is not contained in any finitely generated variety; we describe the bottom of the lattice of its subvarieties.
\end{abstract}

\section{Introduction AND PRELIMINARIES}

By a conservative groupoid, we mean an algebra $(A, \cdot)$ in which $x \cdot y \in$ $\{x, y\}$ for all $x, y \in A$. For such an algebra, we have a reflexive binary relation $R$ on $A$ defined by $R(x, y) \leftrightarrow x \cdot y=x$. The structure $(A, R)$ is definitionally equivalent with $(A, \cdot)$. We call $(A, \cdot)$ the order algebra of $(A, R)$ if $(A, R)$ is an ordered set-i.e., if $R$ is reflexive, transitive and antisymmetric. In case $R$ is a quasi-order on $A$ (or an equivalence relation on $A$ ), i.e., if $R$ is reflexive and transitive (or respectively, reflexive and transitive and symmetric), we call $(A, \cdot)$ the quasi-order algebra of $(A, R)$ (or respectively, the equivalence algebra of $(A, R)$ ).

In [3] we began an investigation of the variety $\mathcal{P}$ generated by order algebras. In the present paper we investigate the variety in more detail. We will prove that the variety is not finitely based, answering a question raised in [3]. We will find a lower bound and an asymptotically close upper bound for both the free spectrum and the G-spectrum of $\mathcal{P}$; it will follow that $\mathcal{P}$ is not contained in any finitely generated variety. We will describe the bottom of the lattice of subvarieties of $\mathcal{P}$.

The reader is referred to [9] for the basics of universal algebra and equational theory. We will work mostly with groupoids, i.e., algebras with a single operation, for which we use multiplicative notation. In order to avoid too many parentheses, we adopt the following convention: $a_{1} a_{2} \cdots a_{n}$ stands for $\left(\left(\left(a_{1} a_{2}\right) a_{3}\right) \cdots\right) a_{n}$, and $a \cdot b c$ stands for $a(b c)$. Also, for example, $a b \cdot c d \cdot e f=((a b)(c d))(e f)$.

This material is based upon work supported by the Grant Agency of the Czech Republic, grant no. 201/99/0263, by the South African National Research Foundation, and by the National Science Foundation under grants no. DMS 9596043, DMS 9941276, and DMS 9971352. 
This paper is also related to our previous papers [5], [7] and [6], in which similar questions have been answered for the variety generated by tournaments and for the variety generated by equivalence algebras. Algebraic treatment of oriented graphs (or reflexive binary relations) was started in the paper [10]. Also, [11] is an earlier paper on order algebras.

For any $n \geq 1$, let $\mathcal{P}_{n}$ denote the variety generated by all $n$-element order algebras, and let $\mathcal{P}^{n}$ denote the variety determined by the at most $n$-variable equations of order algebras. So, $\mathcal{P}_{n} \subseteq \mathcal{P}_{n+1} \subseteq \mathcal{P} \subseteq \mathcal{P}^{n+1} \subseteq \mathcal{P}^{n}$ for all $n$. It is easy to see that the free algebra on $n$ generators in $\mathcal{P}$ is the free algebra on $n$ generators in $\mathcal{P}_{n}$, as well as in $\mathcal{P}^{n}$. In [3] we give a description of the free algebra on three generators in $\mathcal{P}$ (it has 21 elements), and we find an independent base for the equations of the variety $\mathcal{P}^{3}$. The base consists of the following five equations:

(E1) $x x=x$,

(E2) $x y \cdot x=y x$,

(E3) $x y \cdot y=x y$,

(E4) $x(x y \cdot z)=x \cdot y z$

(E5) $(x y \cdot z) y=x z \cdot y$.

We will make heavy use of these equations. The following are their most frequently used consequences:

(E6) $x \cdot x y=x y$

(E7) $x y \cdot y z=x y \cdot z$,

(E8) $\quad(x \cdot y z) z=x \cdot y z$,

(E9) $(x \cdot y z) y=x z \cdot y$,

(E10) $x y \cdot z y=x \cdot z y$,

(E11) $x \cdot y x=y x$.

They can be proved as follows.

$$
\begin{aligned}
(\mathrm{E} 6): & x \cdot x y & ={ }_{(\mathrm{E} 3)} x \cdot x y y={ }_{(\mathrm{E} 4)} x \cdot y y={ }_{(\mathrm{E} 1)} x y \\
(\mathrm{E} 7): & x y \cdot y z & ={ }_{(\mathrm{E} 4)} x y \cdot(x y y) z={ }_{(\mathrm{E} 3)} x y \cdot x y z={ }_{(\mathrm{E} 6)} x y \cdot z \\
(\mathrm{E} 8): & x \cdot y z & ={ }_{(\mathrm{E} 3)}(x \cdot y z) \cdot y z=_{(\mathrm{E} 3)}(x \cdot y z) \cdot y z z={ }_{(\mathrm{E} 7)}(x \cdot y z) z \\
(\mathrm{E} 9): & (x \cdot y z) y & ={ }_{(\mathrm{E} 5)}(x y \cdot y z) y={ }_{(\mathrm{E} 7)} x y z y={ }_{(\mathrm{E} 5)} x z \cdot y \\
(\mathrm{E} 10): & x y \cdot z y & ={ }_{(\mathrm{E} 8)}(x y \cdot z y) y={ }_{(\mathrm{E} 5)}(x \cdot z y) y={ }_{(\mathrm{E} 8)} x \cdot z y \\
(\mathrm{E} 11): & x \cdot y x & ={ }_{(\mathrm{E} 2)}(y x \cdot x) \cdot y x=_{(\mathrm{E} 3)} y x \cdot y x=_{(\mathrm{E} 1)} y x .
\end{aligned}
$$

A more comprehensive collection of three-variable consequences of (E1) - (E5) with proofs is given in [3].

It is also possible to describe the normal form, with respect to the equational theory based on (E1) through (E5), of a general term in three variables. The following algorithm is based on the result of [3]. Let $t$ be a term in precisely three variables. The normal form of $t$ should be a linear term (i.e., a term containing no variable more than once), in the same variables. There are twenty-one candidates for this normal form, as there 
are twenty-one linear terms in three specified variables. Let us arrange the variables of an arbitrary term $u$ into a sequence of pairwise different variables $x_{1}, \ldots, x_{k}$ in the following standard way. Let $x_{k}$ be the last variable in $u$, let $x_{k-1}$ be the last variable different from $x_{k}$ in $u$, let $x_{k-2}$ be the last variable different from both $x_{k}$ and $x_{k-1}$ in $u$, and so on. Now both sides of an arbitrary equation that is a consequence of (E1)-(E5) must have the same sets of variables, with the same arrangement in the above standard sense. Suppose that the given term $t$ has its three variables arranged into the sequence $x, y, z$. Since the normal form must have its variables arranged in the same way, the search is narrowed to just two candidates: the terms $(x y) z$ and $x(y z)$. Now evaluate the term $t$ in the order algebra with three elements $a, b, c$ and a single covering $a<b$, under the interpretation $x \mapsto a$, $y \mapsto c, z \mapsto b$. The obtained value is always either $a$ or $b$. If it is $a$, the normal form of $t$ is $x(y z)$; if it is $b$, the normal form is $(x y) z$.

For any algebra $\mathbf{A}=(A, \cdot) \in \mathcal{P}^{3}$ and $a, b$ in $A$, we define $a \leq b$ if and only if $a b=a$. This is a partial order on $A$ (as follows easily from equations (E1), (E2) and (E8).

Lemma 0.1. The following hold in $\mathcal{P}^{3}$ :

(1) $x \leq y$ implies $x z \leq y z$;

(2) always $x y \leq y$; if $x y \leq x$, then $x y=y x$ is the meet of $x$ and $y$;

(3) $x \cdot y z \leq x y \cdot z$;

(4) $x_{1} x_{2} \cdots x_{i-1} y x_{i+1} \cdots x_{n} y=x_{1} x_{2} \cdots x_{i-1} x_{i+1} \cdots x_{n} y$ for $1 \leq i \leq n$;

(5) $x y_{1} \cdots y_{n} \leq y_{1} \cdots y_{n}$ for any $n \geq 1$;

(6) $x\left(x y_{1} \cdots y_{n}\right)=x\left(y_{1} \cdots y_{n}\right)$ for any $n \geq 1$;

(7) $t=t x$ for any term $t$ with the rightmost variable $x$.

Proof. (1) $x z \cdot y z=x \cdot y z=x(x y \cdot z)=x \cdot x z=x z$.

(2) If $z \leq x$ and $z \leq y$, then $z \cdot x y=z(z x \cdot y)=z \cdot z y=z z=z$.

(3) $(x \cdot y z)(x y \cdot z)={ }_{(4)} x(x y \cdot z) \cdot(x y \cdot z)={ }_{(3)} x(x y \cdot z)={ }_{(4)} x \cdot y z$.

(4) Apply $x y z y=x z y$ several times to insert $y$ 's between the $x$ 's from right to the left and then remove them in reverse order.

(5) By induction on $n$, using (2).

(6) By induction on $n$, using (E4).

(7) We need to prove $t \leq x$. This can be done by induction on $t$, using $p q \leq q \leq x$.

For an order algebra $\mathbf{P}$ and a triple $a, b, c$ of elements of $\mathbf{P}$, we denote by $\mathbf{P}[a b=c]$ the algebra that differs from $\mathbf{P}$ only in setting $a b=c$. We will also need the following from [3]:

Lemma 0.2. Let $\mathbf{P}$ be an ordered set and $a, b, c$ be a triple of pairwise different elements of $\mathbf{P}$ such that $a$ is incomparable with $b$. The algebra $\mathbf{P}[a b=c]$ belongs to $\mathcal{P}^{3}$ if and only if the following conditions are satisfied:

(1) $c<b$ and $c \not \leq a$,

(2) for any $x \in P, x>c$ implies $x \geq b$, 


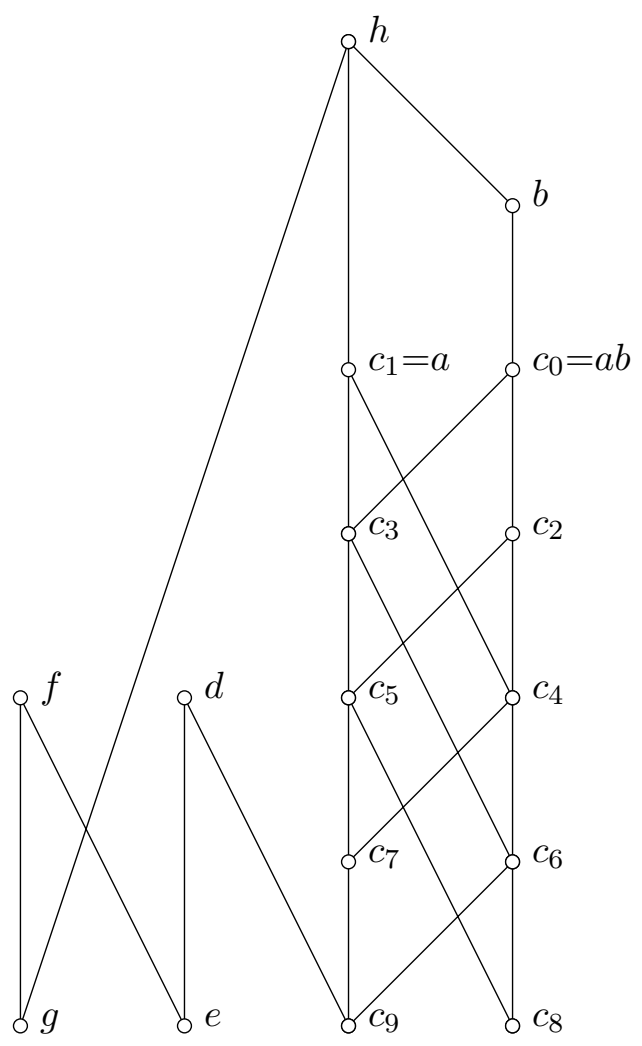

Fig. 1

(3) for any $x \in P, x<a$ implies $x<c$,

(4) for any $x \in P, x>a$ if and only if $x>b$.

\section{The Variety $\mathcal{P}$ is not Finitely Based}

For every $n \geq 3$ let $\mathbf{Q}_{n}^{\prime}$ be the poset with $n+7$ elements $b, c_{0}, \ldots, c_{n}, d, e$, $f, g, h$ (put $a=c_{1}$ ) and order relation described by the following coverings:

$$
\begin{aligned}
& c_{i+2}<c_{i} \text { for } i=0, \ldots, n-2, \\
& c_{i+3}<c_{i} \text { for } i=0, \ldots, n-3, \\
& a<h, \quad c_{0}<b<h, \quad c_{n}<d, \quad e<d, \quad e<f, \quad g<f, \quad g<h .
\end{aligned}
$$

Denote by $\mathbf{Q}_{n}$ the algebra $\mathbf{Q}_{n}^{\prime}\left[a b=c_{0}\right]$. (For example, $\mathbf{Q}_{9}$ is shown in Fig. 1.) 
Let us fix $n+6$ variables $B, C_{1}, \ldots, C_{n}, D, E, F, G, H$ (put $A=C_{1}$ ). For any term $X$ define terms $t_{i}=t_{i}(X)(i=2, \ldots, n, E, F, G, H, B)$ as follows:

$$
\begin{aligned}
& t_{2}=C_{2}(A B \cdot X) \cdot A B, \\
& t_{i}=C_{i} t_{i-1} \cdot C_{i-2} \quad(i=3, \ldots, n,) \\
& t_{E}=C_{n}\left(E \cdot t_{n} D\right), \\
& t_{F}=t_{E} \cdot C_{n} F, \\
& t_{G}=E \cdot G t_{F}, \\
& t_{H}=t_{G} \cdot E H, \\
& t_{B}=G\left(A B \cdot t_{H}\right) \cdot B .
\end{aligned}
$$

Lemma 1.1. $Q_{n}$ does not satisfy the equation $t_{B}(A)=t_{B}(B)$.

Proof. Consider the interpretation $B \rightarrow b, C_{i} \rightarrow c_{i}, D \rightarrow d, E \rightarrow e, F \rightarrow f$, $G \rightarrow g, H \rightarrow h$. If $n$ is odd, then $t_{B}(A)$ evaluates to $b$, while $t_{B}(B)$ evaluates to $c_{0}$. If $n$ is even, then $t_{B}(A)$ evaluates to $c_{0}$, while $t_{B}(B)$ evaluates to $b$.

Lemma 1.2. The equation $t_{B}(A)=t_{B}(B)$ is satisfied in any quasi-ordered set.

Proof. Suppose that in a given quasi-ordered set, there is an interpretation of the variables $A, B, C_{1}, \ldots, C_{n}, D, E, F, G, H$ under which the terms $t_{B}(A)$ and $t_{B}(B)$ evaluate to different elements. Denote by $a, b, c_{1}, \ldots, c_{n}, d, e, f$, $g, h$ the interpreted elements (we have $c_{1}=a$ ) and for each $i$ denote by $t_{i}(a)$ and $t_{i}(b)$ the values of $t_{i}(A)$ and $t_{i}(B)$ under that interpretation. Since $t_{B}(a) \neq t_{B}(b)$, we have $t_{i}(a) \neq t_{i}(b)$ for all $i$.

If $a b=a$, then $t_{2}(a)=t_{2}(b)=c_{2} a$, a contradiction. So, we have $a \not \leq b$. If $b a=b$, then $t_{2}(a)=t_{2}(b)=c_{2} b$, a contradiction. Hence $b \not \leq a$. We see that $a \| b$ (by which we mean $a \not \leq b$ and $b \not \leq a)$. We have $t_{2}(a)=c_{2} a \cdot b$ and $t_{2}(b)=c_{2} b$. Since these are different elements, we get $c_{2} a=a \neq c_{2}$ and $c_{2} b=c_{2} \neq b$. Put $c_{0}=a b=b$. We have $c_{1}\left\|c_{0}, c_{2}\right\| c_{1}$ (since $c_{1} \leq c_{2}$ would imply $c_{1} \leq c_{0}$ by transitivity), $c_{2}<c_{0}$ and $\left\{t_{2}(a), t_{2}(b)\right\}=\left\{c_{0}, c_{2}\right\}$.

Let us prove by induction on $i=2, \ldots, n$ that $c_{i-1}\left\|c_{i-2}, c_{i}\right\| c_{i-1}, c_{i}<c_{i-2}$ and $\left\{t_{i}(a), t_{i}(b)\right\}=\left\{c_{i-2}, c_{i}\right\}$. This has been verified for $i=2$. Now suppose that the assertion has been proved for some $i<n$. If $c_{i+1} c_{i}=$ $c_{i+1}$, then $c_{i+1} \leq c_{i} \leq c_{i-2}$ imply $c_{i+1} c_{i-2}=c_{i+1}=c_{i+1} c_{i}$ by transitivity, so that $t_{i+1}(a)=t_{i+1}(b)$, a contradiction. Hence $c_{i+1} \not \leq c_{i}$. We get $\left\{t_{i+1}(a), t_{i+1}(b)\right\}=\left\{c_{i+1} c_{i-2} \cdot c_{i-1}, c_{i-1}\right\}$. This forces $c_{i+1} c_{i-2}=c_{i+1}$ and $c_{i+1} c_{i-1}=c_{i+1} \neq c_{i-1}$, so that $c_{i+1}<c_{i-1}$ and $\left\{t_{i+1}(a), t_{i+1}(b)\right\}=$ $\left\{c_{i+1}, c_{i-1}\right\}$. We also have $c_{i+1} \| c_{i}$, since $c_{i} \leq c_{i+1}$ would imply $c_{i} \leq c_{i-1}$ by transitivity.

In particular, $c_{n}<c_{n-2}$ and $\left\{t_{n}(a), t_{n}(b)\right\}=\left\{c_{n-2}, c_{n}\right\}$. Now we are going to prove that $e \| c_{n}$ and $\left\{t_{E}(a), t_{E}(b)\right\}=\left\{e, c_{n}\right\}$. Consider two cases.

Case 1: $c_{n-2} \leq d$. Then $t_{E}(a) \neq t_{E}(b)$ means $c_{n} \cdot e c_{n-2} \neq c_{n} \cdot e c_{n}$, from which we get $e \not \leq c_{n}, e<c_{n-2}, c_{n} \not \leq e$ and $\left\{c_{n} \cdot e c_{n-2}, c_{n} \cdot e c_{n}\right\}=\left\{e, c_{n}\right\}$. 
Case 2: $c_{n-2} \not \leq d$. Then $t_{E}(a) \neq t_{E}(b)$ means $c_{n}(e d) \neq c_{n}\left(e \cdot c_{n} d\right)$, from which we get $c_{n}<d$ and $e d \neq e c_{n}$. Thus $c_{n} \cdot e d \neq c_{n} \cdot e c_{n}$. We cannot have $e \leq c_{n}$ (this would imply $e \leq d$ and then $e d=e c_{n}$ ), and we cannot have both $e d=d$ and $e c_{n}=c_{n}$ (this would imply $c_{n} \cdot e d=c_{n} \cdot e c_{n}$ ). The only remaining possibility is that $e<d$ and $e \not \leq c_{n}$. Now $t_{E}(a) \neq t_{E}(b)$ means $c_{n} e \neq c_{n}$, so that $c_{n} e=e \neq c_{n}$.

We have obtained $e \| c_{n}$ and $\left\{t_{E}(a), t_{E}(b)\right\}=\left\{e, c_{n}\right\}$ in both cases. We have $\left\{t_{F}(a), t_{F}(b)\right\}=\left\{e \cdot c_{n} f, c_{n} \cdot c_{n} f\right\}$. If $c_{n} f=c_{n}$, this would be a oneelement set $\left\{c_{n}\right\}$. Hence $c_{n} \not \leq f, e<f$ and $\left\{t_{F}(a), t_{F}(b)\right\}=\{e, f\}$.

Now from $t_{G}(a) \neq t_{G}(b)$ we easily obtain that $g \| e, g<f$ and $\left\{t_{G}(a)\right.$, $\left.t_{G}(b)\right\}=\{e, g\}$. Then from $t_{H}(a) \neq t_{H}(b)$ we obtain $e \not \leq h, g<h$ and $\left\{t_{H}(a), t_{H}(b)\right\}=\{g, h\}$.

Finally, $t_{B}(a) \neq t_{B}(b)$ says $(g \cdot b g) b \neq(g \cdot b h) b$. If $b \leq g$, then also $b \leq h$ and both sides are equal to $b$. Hence $b g=g$. Consequently, $(g \cdot b g) b=g b$. But also $(g \cdot b h) b=g b$ : this can be easily checked by considering two cases, corresponding to either $b h=b$ or $b h=h$. So we get $t_{B}(a)=t_{B}(b)$, a contradiction.

Lemma 1.3. The algebra $\mathbf{Q}_{n},(n \geq 3)$, is generated by $n+6$ elements. Every subalgebra of $\mathbf{Q}_{n}$ generated by at most $n+1$ elements is either an order algebra or is isomorphic to a subalgebra of the direct product of two order algebras. Consequently, $\mathbf{Q}_{n}$ satisfies all the equations of $\mathcal{P}$ in at most $n+1$ variables.

Proof. A subalgebra generated by at most $n+1$ elements cannot contain all the elements $a, b, c_{2}, \ldots, c_{n}, d$. Put $c_{n+1}=d$. By removing either $a$ or $b$ from $\mathbf{Q}_{n}$, we obtain an order subalgebra. By removing an element $c_{i}$ with $i \geq 2$, we obtain a subalgebra with two congruences intersecting to the identity, one relating $b$ with $c_{0}$ and the second relating the elements $c_{0}, \ldots, c_{i-1}$, such that both factors are order algebras.

Theorem 1.4. The variety $\mathcal{P}$ is not finitely based. Moreover, there is no finitely based variety containing $\mathcal{P}$ and contained in the variety generated by quasi-ordered sets.

Proof. It is a combination of lemmas 1.1, 1.2 and 1.3.

Theorem 1.5. The equation $x y z x=x(z y) z x$ is satisfied in all order algebras and also in all equivalence algebras, but not in the three-element quasiorder algebra with one greatest and two least elements. Consequently, the join of $\mathcal{P}$ with the variety generated by equivalence algebras is a proper subvariety of the variety generated by quasi-order algebras.

Proof. It is easy to check all the statements.

\section{The Variety is not Finitely Generated}

Theorem 2.1. Let $\mathbf{F}$ be the order algebra of a finite crown or fence, with at least three maximal elements. If $\mathbf{F}$ lies in the variety generated by a family 
$\mathcal{U}$ of algebras in $\mathcal{P}$, then $\mathbf{F}$ is a homomorphic image of a subalgebra of an algebra from $\mathcal{U}$.

Proof. We can assume that we have a surjective homomorphism $\varphi: \mathbf{S} \rightarrow \mathbf{F}$, where $\mathbf{S}$ is a subalgebra of $\mathbf{P}_{1} \times \cdots \times \mathbf{P}_{n}, n$ finite, and all $\mathbf{P}_{i}$ are finite subalgebras of algebras in $\mathcal{U}$. Choose and hold fixed five distinct elements $b_{0}, b_{1}, b_{2}, a_{0}, a_{1} \in F$ with $b_{0}>a_{0}<b_{1}>a_{1}<b_{2}$. For $f, g \in S$, denote by $[|f=g|]$ the equalizer set $\{i: f(i)=g(i)\}$.

Claim 1: Suppose we have an element $\beta_{1}$ which is minimal in $\varphi^{-1}\left(b_{1}\right)$, $\beta_{2}$ minimal in $\varphi^{-1}\left(b_{2}\right)$, and elements $\alpha_{0} \in \varphi^{-1}\left(a_{0}\right), \delta \in \varphi^{-1}\left(a_{1}\right)$, and $\gamma \in$ $\varphi^{-1}\left(b_{1}\right)$. Then where $\tau=\left(\delta \beta_{1}\right) \beta_{2}$, we have $\tau \in \varphi^{-1}\left(a_{1}\right)$ and $\left[\left|\alpha_{0}=\beta_{1}\right|\right] \cup$ $[|\delta=\gamma|] \subseteq\left[\left|\tau=\beta_{2}\right|\right]$.

Indeed, suppose $\alpha_{0}(i)=\beta_{1}(i)$ for some $i$. Note that the element $\left(\delta \alpha_{0}\right) \beta_{2}$ belongs to $\varphi^{-1}\left(b_{2}\right)$ and is $\leq \beta_{2}$, and so $\left(\delta \alpha_{0}\right) \beta_{2}=\beta_{2}$. Then $\tau(i)=$ $\left(\delta(i) \alpha_{0}(i)\right) \beta_{2}(i)=\beta_{2}(i)$. Next, suppose that $\delta(i)=\gamma(i)$. Note that by minimality of $\beta_{2}$, we have $\left(\gamma \beta_{1}\right) \beta_{2}=\beta_{2}$. Thus $\tau(i)=\left(\gamma(i) \beta_{1}(i)\right) \beta_{2}(i)=\beta_{2}(i)$.

Claim 2: For any two distinct elements $x, y$ of $\mathbf{F}$, there are polynomial functions $p$ and $q$ of $\mathbf{F}$ such that $\{p(x), p(y)\}=\left\{a_{0}, b_{1}\right\}$ and $\{q(x), q(y)\}=$ $\left\{a_{1}, b_{1}\right\}$. The proof is easy and is left to the reader.

Claim 3: If $f, g \in S$ and $\varphi(f) \neq \varphi(g)$, then there exist a minimal element $\beta_{1} \in \varphi^{-1}\left(b_{1}\right)$ and elements $\alpha_{0} \in \varphi^{-1}\left(a_{0}\right), \gamma \in \varphi^{-1}\left(b_{1}\right), \delta \in \varphi^{-1}\left(a_{1}\right)$ such that $[|f=g|] \subseteq\left[\left|\alpha_{0}=\beta_{1}\right|\right]$ and $[|f=g|] \subseteq[|\delta=\gamma|]$.

For the proof, use polynomials $p$ and $q$, as in Claim 2, to move $\{\varphi(f), \varphi(g)\}$ to $\left\{a_{0}, b_{1}\right\}$ and $\left\{a_{1}, b_{1}\right\}$. Without loss of generality, we can assume that $p(\varphi(f))=a_{0}$ and $p(\varphi(g))=b_{1}$. There is a polynomial $p^{\prime}$ of $\mathbf{S}$ such that $\varphi\left(p^{\prime}(x)\right)=p(\varphi(x))$ for all $x \in S$. Let $\beta_{1}$ be minimal in $\varphi^{-1}\left(b_{1}\right)$. Then $p^{\prime}(g) \beta_{1}=\beta_{1}$ and we can take $\alpha_{0}$ to be $p^{\prime}(f) \beta_{1}$. Then clearly $[|f=g|] \subseteq$ $\left[\left|\alpha_{0}=\beta_{1}\right|\right]$. A similar construction gives $\delta$ and $\gamma$.

Claim 4: If $f, g, h, k$ are elements of $S$ such that $\varphi(f) \neq \varphi(g)$ and $\varphi(h) \neq$ $\varphi(k)$, then there exist elements $u, v \in S$ such that $\varphi(u) \neq \varphi(v)$ and $[\mid f=$ $g \mid] \cup[|h=k|] \subseteq[|u=v|]$.

Claim 4 is obtained easily by applying Claims 1 and 3. By repeatedly applying Claim 4, we obtain two elements $u, v \in S$ such that $\varphi(u) \neq \varphi(v)$ and $[|f=g|] \subseteq[|u=v|]$ for all $f, g \in S$ with $\varphi(f) \neq \varphi(g)$. Since $u \neq v$, there exists an $i \notin[|u=v|]$, and hence $f(i) \neq g(i)$ for all $f, g \in S$ with $\varphi(f) \neq \varphi(g)$. For this $i$, it is easy to see that $F$ is a homomorphic image of a subalgebra of $P_{i}$.

Corollary 2.2. The variety $\mathcal{P}$ is not finitely generated.

Theorem 2.3. The variety $\mathcal{P}$ has uncountably many subvarieties. The lattice of subvarieties of $\mathcal{P}$ contains a subset, order isomorphic to the lattice of all subsets of a countably infinite set.

Proof. For any subset $S$ of $\{3,4, \ldots\}$ denote by $\mathcal{V}_{S}$ the variety generated by the crowns $\mathbf{C}$ such that the number of maximal elements of $\mathbf{C}$ belongs to $S$. It follows from 2.1 that these varieties are pairwise distinct. (Observe that 
order algebras corresponding to crowns are simple and an order algebra of a crown of cardinality $k$ is not a homomorphic image of a subalgebra of one of cardinality $n$ for $n \neq k$.)

\section{The Bottom of the Lattice of Subvarieties}

Denote by

$\mathcal{P}_{\mathrm{S}}$ the variety of semilattices,

$\mathcal{P}_{\mathrm{A}}$ the variety of antichains (groupoids satisfying $x y=y$ ),

$\mathcal{P}_{\vee}$ the variety generated by the order algebra $\mathbf{O}_{\vee}$ with elements $a, b, c$ and coverings $a<b$ and $a<c$,

$\mathcal{P}_{\wedge} \quad$ the variety generated by the order algebra $\mathbf{O}_{\wedge}$ with elements $a, b, c$ and coverings $a<c$ and $b<c$,

$\mathcal{P}_{2+1}$ the variety generated by the order algebra $\mathbf{O}_{2+1}$ with elements $a, b, c$ and a single covering $a<b$.

Theorem 3.1. The variety $\mathcal{P}_{\wedge}$ is the intersection of $\mathcal{P}^{3}$ with the variety of semigroups. It is just the variety of idempotent semigroups satisfying $x y x=y x$. It has three proper nontrivial subvarieties: $\mathcal{P}_{\mathrm{S}}, \mathcal{P}_{\mathrm{A}}$, and their join, $\mathcal{P}_{\vee}$. The variety $\mathcal{P}_{\vee}$ is the variety of idempotent semigroups satisfying $x y z=y x z$; also, it is the intersection of $\mathcal{P}^{3}$ with the variety of medial groupoids (groupoids satisfying $x y \cdot z u=x z \cdot y u$ ).

Proof. It is easy to check that the intersection $\mathcal{V}$ of $\mathcal{P}^{3}$ with the variety of semigroups is the variety of idempotent semigroups satisfying $x y x=$ $y x$. The lattice of varieties of idempotent semigroups has been described in [1]. From the description it follows that the lattice of subvarieties of $\mathcal{V}$ has precisely five elements: the atoms are the varieties $\mathcal{P}_{\mathrm{S}}$ and $\mathcal{P}_{\mathrm{A}}$, and the coatom is the variety of idempotent semigroups satisfying $x y z=y x z$. One can easily check that $\mathbf{O}_{\wedge}$ belongs to $\mathcal{V}$, but does not satisfy $x y z=y x z$. It follows that $\mathcal{V}=\mathcal{P}_{\wedge}$. Now it is easy to see that the largest proper subvariety of $\mathcal{P}_{\wedge}$ is $\mathcal{P}_{\vee}$. Medial groupoids belonging to $\mathcal{P}^{3}$ are associative: $x y \cdot z=$ $x y \cdot z z=x z \cdot y z=x \cdot y z$.

It has been proved in [2] (for a more complete proof see [4]) that every semigroup from $\mathcal{P}_{\wedge}$ can be embedded into a subdirectly irreducible semigroup in $\mathcal{P}_{\wedge}$. Hence the variety $\mathcal{P}_{\wedge}$ is residually large. It will follow easily from 3.3 and 3.7 that the variety $\mathcal{P}_{2+1}$ is also residually large.

Theorem 3.2. An algebra $\mathbf{A} \in \mathcal{P}^{3}$ belongs to $\mathcal{P}_{\wedge}$ if and only if it does not contain a three-element subalgebra isomorphic to $\mathbf{O}_{2+1}$.

Proof. For the direct implication, it is sufficient to check that $\mathbf{O}_{2+1}$ is not a semigroup. Now let $\mathbf{A}$ be a non-associative algebra in $\mathcal{P}^{3}$. By 0.1(3), A contains three elements $a, b, c$ such that $a \cdot b c<a b \cdot c$. Put $a^{\prime}=a \cdot b c$, $b^{\prime}=a b \cdot c$ and $c^{\prime}=a c \cdot b$. One can easily verify that $a^{\prime} c^{\prime}=b^{\prime} c^{\prime}=c^{\prime}, c^{\prime} a^{\prime}=a^{\prime}$ and $c^{\prime} b^{\prime}=b^{\prime}$. Consequently, $c^{\prime}$ is different from both $a^{\prime}$ and $b^{\prime}$ and $\left\{a^{\prime}, b^{\prime}, c^{\prime}\right\}$ is a subalgebra isomorphic to $\mathbf{O}_{2+1}$. 
Theorem 3.3. The following are equivalent for an algebra $\mathbf{A} \in \mathcal{P}^{3}$ :

(1) A satisfies $x \cdot y z=y \cdot x z$;

(2) A does not contain a three-element subalgebra isomorphic to $\mathbf{O}_{\wedge}$;

(3) for any $a \in A$, the set $\{x \in A: x \leq a\}$ is a subsemilattice.

Proof. (1) implies (3): If $x \leq z$ and $y \leq z$, then $x y \cdot x=x y \cdot x z=y \cdot x z=$ $x \cdot y z=x y$, so that $x y \leq x$ and then $x y=y x$ is the meet of $x$ and $y$ according to $0.1(2)$.

Clearly, (3) implies (2). It remains to prove that (2) implies (1). Let $\mathbf{A} \in \mathcal{P}^{3}$ contain elements $a, b, c$ such that $a \cdot b c \neq b \cdot a c$. It is easy to see that $\{a \cdot b c, b \cdot a c, c\}$ is a subalgebra isomorphic to $\mathbf{O}_{\wedge}$.

Let us denote by $\Gamma_{0}$ the equational theory based on the five equations (E1) through (E5), and by $\Gamma_{1}$ the equational theory based on $\Gamma_{0}$ and $x \cdot y z=y \cdot x z$. We are going to prove in 3.7 that $\Gamma_{1}$ is the equational theory of $\mathbf{O}_{2+1}$. We need some lemmas.

Lemma 3.4. The following equations belong to $\Gamma_{1}$ :

(1) $x(y z u)(y u z)=x(y u) z$;

(2) $x z(y z u)=x(y z) u$;

(3) $x(y u) z u=x(y z) u$;

(4) $(a y)(b y) x=(a y x)(b y x)$;

(5) $y u(z x) \leq y z u x$;

(6) $(y u x)(z v x) \leq y z u v x$;

(7) $\left(y_{k} \cdots y_{1} x\right)\left(y_{k+1} z_{1} \cdots z_{m} x\right) \leq y_{k+1} y_{k} \cdots y_{1} x$ for any $\left\{z_{1}, \cdots, z_{m}\right\} \subseteq$ $\left\{y_{1}, \cdots, y_{k}\right\}$.

Proof. We will try to indicate in subscripts for each step in the following derivations, which equation or which lemma has been used. Here $(*)$ stands for $x \cdot y z=y \cdot x z$. We have

$$
\begin{aligned}
& x(y z u)(y u z)=_{(\mathrm{E} 5)} x(y z u)(y z u z)={ }_{(\mathrm{E} 7)} x(y z u) z=_{(*)}(y z)(x u) z \\
& ={ }_{(\mathrm{E} 5)} y(x u) z={ }_{(*)} x(y u) z \\
& x z(y z u)=_{(\mathrm{E} 4)} x z(x z(y z) u)=_{(\mathrm{E} 10)} x z(x(y z) u)=_{(*)} x(y z)(x z u) \\
& =_{(\mathrm{E} 5)} x(x z u)(y z)(x z u)={ }_{(\mathrm{E} 4)} x(z u)(y z)(x z u) \\
& =_{(*)} y(x(z u) z)(x z u)={ }_{(\mathrm{E} 9)} y(x u z)(x z u)={ }_{(3.4 .1)} y(x z) u \\
& ={ }_{(*)} x(y z) u \\
& x(y u) z u={ }_{(3.4 .2)} x u(y u z) u={ }_{(\mathrm{E} 5)} x(y u z) u={ }_{(*)} y u(x z) u \\
& ={ }_{(\mathrm{E} 5)} y(x z) u={ }_{(*)} x(y z) u \\
& (a y)(b y) x={ }_{(\mathrm{E} 10)} a(b y) x={ }_{(3.4 .2)} a y(b y x)={ }_{(\mathrm{E} 10)}(a y x)(b y x) \\
& y u(z x)={ }_{(\mathrm{E} 5)} y(z x) u(z x)=_{(\mathrm{E} 10)} y(z x) u x(z x) \leq_{(3.3 .3)} y(z x) u x \\
& { }_{(3.4 .3)} y(z u) x \leq_{(0.1 .1,0.1 .3)} y z u x \\
& (y u x)(z v x)=_{(\mathrm{E} 10)} y u(z v x) \leq_{(0.1 .3)} y u(z v) x \leq_{(3.4 .5,0.1 .1)} y z u v x
\end{aligned}
$$




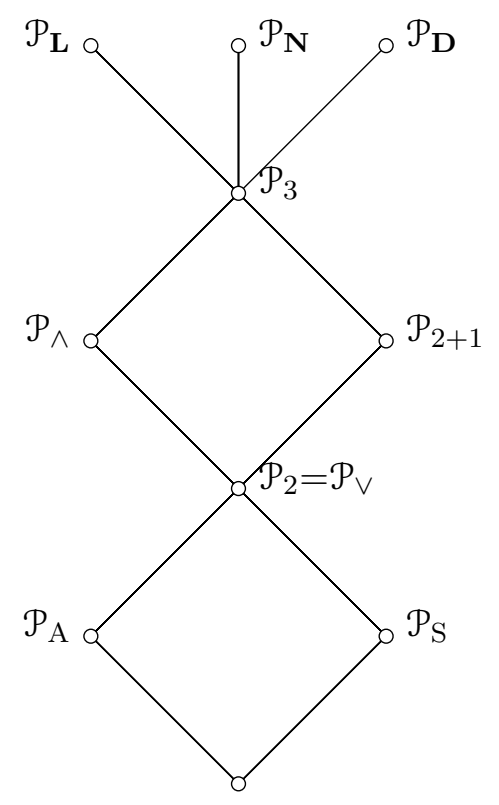

Fig. 2

It remains to prove (7). By 0.1.4, the left side is

$$
\left(x \cdots x y_{k} \cdots y_{1} x\right)\left(y_{k+1} z_{1} \cdots z_{m} x \cdots x\right)
$$

with both blocks of $x$ 's sufficiently long. By repeated applications of (6) we get that this term is $\leq y_{k+1} z_{1} \cdots z_{m} y_{k} \cdots y_{1} x$. Since every $z_{i}$ is identical with some one of $y_{k}, \cdots, y_{1}$, by 0.1 .4 we can successively remove every $z_{i}$, obtaining $y_{k+1} y_{k} \cdots y_{1} x$.

By an $x$-normal constituent (where $x$ is a variable) we mean a term $y_{1} y_{2} \cdots y_{k} x$ where $k \geq 0$ and $y_{1}, \ldots, y_{k}, x$ are pairwise different variables. By an $x$-normal term we will mean a product of $x$-normal constituents containing the same sets of variables. (Notice that the multiplication of $x$-normal constituents, for a fixed $x$, is both commutative and associative with respect to $\Gamma_{1}$.)

Lemma 3.5. With respect to the equational theory based on $\Gamma_{0}$ and $3.4(2)$, every term $t$ is equivalent to an $x$-normal term, where $x$ is the last variable in $t$.

Proof. Since $(x z)(y z \cdot u z)={ }_{(3.4(2))}(x \cdot y z)(u z)={ }_{(\mathrm{E} 11)}(x z \cdot y z)(u z)$, it is easy to see that if $t_{1}, \ldots, t_{n}$ are any terms such that the last variables in $t_{1}, \ldots, t_{n}$ are all equal, then the product $t_{1} \cdots t_{n}$ is associative (does not depend on the arrangement of parentheses). It is not difficult to see that if $a=\left(a_{1} x\right) \cdots\left(a_{n} x\right)$ is a product of $x$-normal constituents and $b=\left(b_{1} y\right) \cdots\left(b_{m} y\right)$ is a product 

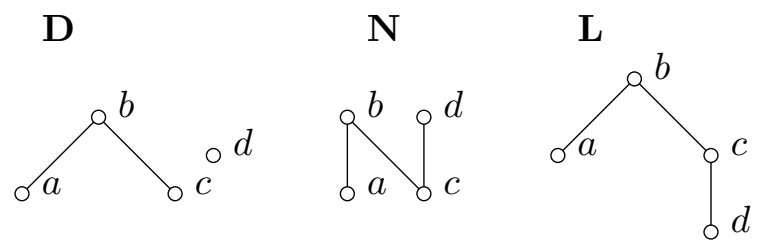

Fig. 3

of $y$-normal constituents, then $a b$ is a product of $y$-normal constituents: $a b$ is equivalent to $\left(a_{1} x y\right) \cdots\left(a_{n} x y\right)\left(b_{1} y\right) \cdots\left(b_{m} y\right)$. (To prove this, we use induction on $n$. If $n=1$, use (E14). If $n \geq 2$, then $\left(a^{\prime} \cdot a_{n} x\right) b={ }_{(3.4(2))}$ $a^{\prime} x \cdot\left(a_{n} x \cdot b\right)=a^{\prime}\left(a_{n} x \cdot b\right)$ and this can be reduced to $n=1$, as $a^{\prime} x=a^{\prime}$.) Now using $0.1(6), 0.1(4)$ and the associativity, we can easily get a product of $x$-normal constituents into the normal form.

Lemma 3.6. Let $w=\left(a_{1} x\right) \cdots\left(a_{n} x\right)$ be an $x$-normal term and let $b x=$ $y_{1} \cdots y_{k} x$ be an $x$-normal constituent. Then $w \leq b x$ is satisfied in $\mathbf{O}_{2+1}$ if and only if for every $1 \leq j \leq k$ there is an $i \in\{1, \ldots, n\}$ such that $a_{i}$ can be written as $z_{1} \cdots z_{s} \cdots z_{m}$ for some $1 \leq s \leq m$, where $z_{s}=y_{j}$ and $\left\{z_{s+1}, \ldots, z_{m}\right\} \subseteq\left\{y_{j+1}, \ldots, y_{k}\right\}$.

Proof. Denote the elements of $\mathbf{O}_{2+1}$ by 0,1 , and $c$ (where $0<1$ ). If $w \leq b x$ is satisfied, then consider the interpretation $y_{j} \mapsto 0, y_{j^{\prime}} \mapsto 1$ for any $j^{\prime}>j$, $x \mapsto 1$ and $v \mapsto c$ for any other variable $v$. Under this interpretation, $b x$ evaluates to 0 , so that $w$ also must evaluate to 0 . From this it follows that for every $j$ there exists an $i$ as above. The converse is easy.

Theorem 3.7. The equations (E1) through (E5), together with the equation $x \cdot y z=y \cdot x z$, constitute a base for the equational theory of $\mathbf{O}_{2+1}$.

Proof. In view of 3.5 , it is sufficient to show that if $w=\left(a_{1} x\right) \cdots\left(a_{n} x\right)$ is an $x$-normal term and $b x=y_{m} \cdots y_{1} x$ is an $x$-normal constituent such that $w \leq b x$ is satisfied in $\mathbf{O}_{2+1}$, then $w \leq b x$ belongs to $\Gamma_{1}$. We will prove by induction on $k$ that $w \leq y_{k} \cdots y_{1} x$ belongs to $\Gamma_{1}$. For $k=0$ it is evident. Suppose we already know that $w \leq y_{k} \cdots y_{1} x$ belongs to $\Gamma_{1}$ for some $k<m$. By 3.6, there is an $i$ such that $a_{i}$ ends with $y_{k+1} z_{1} \cdots z_{m}$, where $m \geq 0$ and $\left\{z_{1}, \ldots, z_{m}\right\} \subseteq\left\{y_{1}, \ldots, y_{k}\right\}$. By 0.1 .5 we have $w \leq a_{i} x \leq y_{k+1} z_{1} \cdots z_{m} x$. Since also $w \leq y_{k} \cdots y_{1} x$, we get $w \leq\left(y_{k} \cdots y_{1} x\right)\left(y_{k+1} z_{1} \cdots z_{m} x\right)$, so that $w \leq y_{k+1} y_{k} \cdots y_{1} x$ by 3.4.7.

Theorem 3.8. The lattice of subvarieties of $\mathcal{P}_{3}$ has seven elements (see Fig. 2). Every subvariety of $\mathcal{P}^{3}$ either contains, or is contained in $\mathcal{P}_{3}$.

Proof. It follows from 3.1, 3.2, 3.3 and 3.7. 


\section{Further Results about the Bottom}

In this section we will prove that the variety $\mathcal{P}_{3}$ has precisely three covers in the lattice of subvarieties of $\mathcal{P}$, and we will find a finite equational base for $\mathcal{P}_{3}$ and for one of these covers. In the derivations of equations we freely make use of simple consequences of the equations (E1)-(E5) and of lemmas 0.1 and 0.2 .

Denote by

$\mathcal{P}_{\mathbf{D}}$ the variety generated by the order algebra $\mathbf{D}$ with elements $a, b, c, d$ and coverings $a<b$ and $c<b$,

$\mathcal{P}_{\mathbf{N}}$ the variety generated by the order algebra $\mathbf{N}$ with elements $a, b, c, d$ and coverings $a<b, c<b$ and $c<d$,

$\mathcal{P}_{\mathbf{L}} \quad$ the variety generated by the order algebra $\mathbf{L}$ with elements $a, b, c, d$ and coverings $a<b$ and $d<c<b$.

Also, consider the following equations:

(e0) $x(y(z w))=x(y w)(z w)$

(e1) $x(z y) u=(x y)(z y u)$

(e2) $(x z)(y z)((y z)(x z) u v)=(y z)(x z)(u v)$

(e3) $x(y z) w z=x(y z w) z$

(e4) $x z(y z w) z w=x z(y z w)$,

(e5) $(v u x)(w z u x)(z x)=(v u x)(w u z x)(z x)$,

(e6) $z x(v u x)(w z u x)(u x)=z x(v u x)(w u z x)(u x)$,

(e7) $z(v u(z u x))=v u(z(u x))$.

Theorem 4.1. A subvariety of $\mathcal{P}$ satisfies $(\mathrm{e} 0)$ if and only if it does not contain the algebra $\mathbf{L}$.

Proof. The equation (e0) can be equivalently written as $(x w)((y w)(z w))=$ $(x w)(y w)(z w)$. From this we can see that a subvariety $\mathcal{V}$ of $\mathcal{P}$ satisfies $(\mathrm{e} 0)$ if and only if for any algebra $\mathbf{A} \in \mathcal{V}$, the down-set of any element of $\mathbf{A}$ is a subsemigroup. According to 3.1 and 3.2, this means that the down-set of any element of $\mathbf{A}$ must not contain $\mathbf{O}_{2+1}$ as a subalgebra. But, clearly, this is equivalent to saying that $\mathbf{A}$ does not contain $\mathbf{L}$.

We denote by $\Gamma_{2}$ the equational theory based on $\Gamma_{0}$ and (e1), and by $\Gamma_{3}$ the equational theory based on $\Gamma_{0},(\mathrm{e} 1)$ and (e2).

Under (e0), the product of any terms with the same last variables is associative. The equation (e1), identical with 3.4(2), is stronger than (e0). According to 3.5 , every term $t$ is $\Gamma_{2}$-equivalent to an $x$-normal term, where $x$ is the last variable in $t$.

Lemma 4.2. $\Gamma_{0}$ contains the equation (e4). Moreover, $\Gamma_{0}$ contains the equations

(e4a) $\quad x(y z w) z=x w(y w z)$,

(e4b) $\quad x w z(y w)=x z(y w)$. 
Proof. We have

$x(y z w) z=x(y z w)(y z w z)=x(y w z w)(y w z)=x w(y w z)$,

$x w z(y w)=x w z w(y w)=x z w(y w)=x z(y w)$,

$x z(y z w) z w=x(y z w) z w={ }_{(\mathrm{e} 4 \mathrm{a})} x w(y w z) w={ }_{(\mathrm{e} 4 \mathrm{a})} x w z(y z w)={ }_{(\mathrm{e} 4 \mathrm{~b})} x z(y z w)$.

Lemma 4.3. Modulo $\Gamma_{0}$, the equation (e3) is equivalent to (e1). In particular, (e3) is contained in $\Gamma_{2}$.

Proof. To derive (e3) from (e1),

$$
x(y z) w z=x z(y z) w z={ }_{(\mathrm{e} 1)} x z(y z w) z={ }_{(\mathrm{e} 4)} x(y z w) z w z=x(y z w) z .
$$

To derive (e1) from (e3),

$$
\begin{aligned}
x(y z) w & =x(y z) z w z w=x(y z) w z w={ }_{(\mathrm{e} 3)} x(y z w) z w \\
& =x z(y z w) z w={ }_{(\mathrm{e} 4 \mathrm{a})} x(y w z) w z w=x(y w z) w={ }_{(\mathrm{e} 4 \mathrm{a})} x z(y z w) .
\end{aligned}
$$

Lemma 4.4. $\Gamma_{2}$ contains the equation (e5).

Proof. We have

$$
\begin{aligned}
(v u x)(w z u x)(z x) & =v u((w z u x)(z x))=v u((w z u)(w z u z x)) \\
& =v u((w z u)(w u z x))=v u(w z u(w u z x) x) \\
& =v u(w u z x u(w u z x))=v u(u(w u z x)) \\
& =v u(w u z x)=v u x((w u z x)(z x)),
\end{aligned}
$$

the last because $b z x \leq z x$.

Lemma 4.5. $\Gamma_{2}$ contains the equation (e7).

Proof. We have

$$
\begin{aligned}
x(y z(x z u)) & =x u(y u z(x u z u))={ }_{(\mathrm{e} 1)} \\
& =x u(y u z(x u z) u) \\
& =x u(y u(x u z) u)={ }_{(\mathrm{e} 1)} \\
& =x u(y u(x u) z u) \\
& =(y u(x u) z u)(x u)(y u(x u) z u) \\
& =y u z u(x u)(y u(x u) z u) \\
& =y u z(x u)(y u z(x u) z u) \\
& =y u z(x u)(z u) \\
& =y u z u(x u)(z u)={ }_{(\mathrm{e} 0)} \\
& =y u z u((x u)(z u)) \\
& =y z(x(z u)) .
\end{aligned}
$$


Lemma 4.6. $\Gamma_{2}$ contains the equation (e6).

Proof. We have

$$
\begin{aligned}
z x(\text { vux })(w u z x)(u x) & =z(v u(w u z x(u x))) \\
& =z(v u(w u z x(w u z x u x))) \\
& =z(v u(w z u x z x(w z u x))) \\
& =z(v u(w z u x z(w z u x))) \\
& =z(v u(z(w z u x)))=v u(z(w z u x)) \\
& =\operatorname{vux}(z x(w z u x))=\operatorname{vux}(z x)(w z u x)(u x) .
\end{aligned}
$$

This shows that (e6) is equivalent to the equation

(E) $\operatorname{vux}(z x)(w z u x)=z x(v u x)(w z u x)$,

since modulo $\Gamma_{0}$, both sides of $(\mathrm{E})$ are $\leq u x$. Since $w z u x=z u x(w z u x)$ and in (E) we can replace $w$ by $z$, it follows that (e6) is equivalent to

$$
\operatorname{vux}(z x)(z u x)=z x(v u x)(z u x),
$$

or to

$$
v u(z(z u x))=z(v u(z u x)),
$$

or to the equation (e7). It remains to apply 4.5

Lemma 4.7. $(a x \cdot b x \cdot c x)((b x \cdot a x \cdot c x) u v)=b x \cdot a x \cdot c x \cdot u v$ belongs to $\Gamma_{3}$.

Proof. We have $a x \cdot b x \cdot c x=(a x \cdot c x)(b x \cdot c x)$ and $b x \cdot a x \cdot c x=(b x \cdot c x)(a x \cdot c x)$, so (e2) can be used.

Lemma 4.8. The equation

$$
\left(y_{i_{1}} \cdots y_{i_{m}} x\right)(z x)\left(y_{1} \cdots y_{n} x\right)=(z x)\left(y_{i_{1}} \cdots y_{i_{m}} x\right)\left(y_{1} \cdots y_{n} x\right)
$$

belongs to $\Gamma_{3}$ whenever $i_{1}, \ldots, i_{m} \in\{1, \ldots, n\}$.

Proof. This is clear for $m=0$. Let us proceed by induction on $m$. Put $j=i_{1}$ and

$$
\begin{aligned}
& p x=y_{i_{1}} \cdots y_{i_{m}} x, \quad q x=y_{i_{2}} \cdots y_{i_{m}} x, \\
& r x=y_{j} \cdots y_{n} x, \quad s x=y_{1} \cdots y_{n} x .
\end{aligned}
$$

By induction, $q x \cdot z x \cdot s x=z x \cdot q x \cdot s x$. Clearly, $p x=p x \cdot q x$ and $s x=$ $s x \cdot r x=r x \cdot s x$. Put

$$
w x=p x \cdot z x \cdot q x, \quad w^{\prime} x=z x \cdot p x \cdot q x .
$$

Clearly, $q x \cdot y_{j} x=p x \cdot y_{j} x$, and we have

$$
\begin{aligned}
w x \cdot y_{j} x \cdot y_{j+1} & =p x \cdot z x \cdot q x \cdot y_{j} x \cdot y_{j+1}=p x \cdot z x \cdot p x \cdot y_{j} x \cdot y_{j+1} \\
& =z x \cdot p x \cdot q x \cdot y_{j} x \cdot y_{j+1}=w^{\prime} x \cdot y_{j} x \cdot y_{j+1} .
\end{aligned}
$$

Then by $(\mathrm{e} 1), w x \cdot\left(y_{j} x \cdot y_{j+1}\right)=w^{\prime} x \cdot\left(y_{j} x \cdot y_{j+1}\right)$. Multiplying this equation by $y_{j+2}$ from the right and then by $w^{\prime} x$ from the left, we get $w x \cdot y_{j} x y_{j+1} y_{j+2}=$ $w^{\prime} x \cdot y_{j} x y_{j+1} y_{j+2}$ by 4.7 and (E4). We can continue similarly, until we get $w x \cdot y_{j} x y_{j+1} \cdots y_{n} x=w^{\prime} x \cdot y_{j} x y_{j+1} \cdots y_{n} x$, so that $w x \cdot r x=w^{\prime} x \cdot r x$. Then 
$w x \cdot r x \cdot s x=w^{\prime} x \cdot r x \cdot s x$, i.e., $w x \cdot s x=w^{\prime} x \cdot s x$. Hence $p x \cdot z x \cdot q x \cdot s x=$ $z x \cdot p x \cdot q x \cdot s x$. From this and from $q x \cdot z x \cdot s x=z x \cdot q x \cdot s x$ (the induction assumption) we get

$$
\begin{aligned}
p x \cdot z x \cdot s x & =p x \cdot q x \cdot z x \cdot s x=p x \cdot z x \cdot q x \cdot s x \\
& =z x \cdot p x \cdot q x \cdot s x=z x \cdot p x \cdot s x,
\end{aligned}
$$

the required equation.

Theorem 4.9. $\Gamma_{3}$ is a base for the equational theory of $\mathcal{P}_{3}$.

Proof. Of course, the equations of $\Gamma_{3}$ are satisfied in all three-element order algebras. It remains to prove that $\left(w, w^{\prime}\right) \in \Gamma_{3}$ whenever $\left(w, w^{\prime}\right)$ is satisfied in $\mathcal{P}_{3}$ and $w, w^{\prime}$ are two normal terms. The two terms must be $x$-normal for the same variable $x$, they must contain the same variables, and they must have the same rightmost constituent. According to 4.8, the order of all constituents but the last can be permuted at will in any $x$-normal term.

Thus $w=\left(\Pi_{i} c_{i}\right) c$ and $w^{\prime}=\left(\Pi_{j} d_{j}\right) c$ where $c=y_{1} \cdots y_{n} x$. Since the rightmost constituents are the same, the equality in $\mathcal{P}_{3}$ means just two things: first, whenever $e^{\prime}=z_{1} \cdots z_{n} x$ is a constituent of $w^{\prime}$ and $1 \leq i \leq n$, then there is a constituent $e$ of $w$, equal to some $c_{j}$ or $c$, and some $1 \leq k \leq n$ such that $e=u_{1} \cdots u_{n} x, u_{k}=z_{i}$ and $\left\{u_{k+1}, \ldots, u_{n}\right\} \subseteq\left\{z_{i+1}, \ldots, z_{n}\right\}$. The other thing is that the symmetric condition with $w, w^{\prime}$ interchanged, holds.

Using the first condition, we shall show that for all $j, w=d_{j} w$ is provable from the equations of $\Gamma_{3}$. Then it follows that

$$
w=\left(\Pi_{j} d_{j}\right)\left(\Pi_{i} c_{i}\right) c=\left(\Pi_{i} c_{i}\right)\left(\Pi_{j} d_{j}\right) c .
$$

The symmetric condition will then yield that $w^{\prime}$ is equal to this product, and so we will be done.

So, put the first condition to work. Let $d_{i}$ be one of the constituents of $w^{\prime}$ other than $c, d_{i}=z_{1} \cdots z_{n} x$. The induction is on $j, 1 \leq j \leq n+1$, with the reverse order on the interval, to prove that $w=\left(z_{j} \cdots z_{n} x\right) w$. The ground step, $j=n+1$, is trivial. Suppose that we have it for $j+1 \leq n+1$, and let us derive it for $j$. Well, just choose a constituent $e$ of $w$ (it may be $e=c$ ) and a $k$ such that $e=u_{1} \cdots u_{n} x, u_{k}=z_{j}$ and $\left\{u_{k+1}, \ldots, u_{n}\right\} \subseteq\left\{z_{j+1}, \ldots, z_{n}\right\}$. By induction, we have

$$
\begin{aligned}
w & =\left(z_{j+1} \cdots z_{n} x\right) w=\left(z_{j+1} \cdots z_{n} x\right) e w \\
& =\left(z_{j+1} \cdots z_{n} x\right)\left(u_{k} \cdots u_{n} x\right) e w \\
& =u_{k} \cdots u_{n} x z_{j+1} \cdots z_{n} x\left(u_{k} \cdots u_{n} x\right) w \\
& =\left(u_{k} z_{j+1} \cdots z_{n} x\right) w=\left(z_{j} \cdots z_{n} x\right) w .
\end{aligned}
$$

Thus our proof is finished.

Theorem 4.10. Let $\mathcal{V}$ be a variety of groupoids satisfying $\Gamma_{0}$ and (e1). Then either $\mathbf{D} \in \mathcal{V}$ or $\mathcal{V} \subseteq \mathcal{P}_{3}$. 
Proof. Let $\mathbf{F}$ be the free algebra in $\mathcal{V}$ generated by $x, y, z, w$. Let

$$
\begin{aligned}
& w_{1}=(x y)(z y) w, \\
& y_{1}=w_{1} y, \\
& x_{1}=(z y)(x y)((x y)(z y) w y), \\
& z_{1}=(x y)(z y)((x y)(z y) w y) .
\end{aligned}
$$

Using the three-variable equations and (e1), we deduce that

$$
\begin{aligned}
& w_{1}=y_{1} w_{1}=x_{1} w_{1}=z_{1} w_{1}, \\
& y_{1}=w_{1} y_{1}, \\
& x_{1}=w_{1} x_{1}=z_{1} x_{1}=x_{1} y_{1}, \\
& z_{1}=w_{1} z_{1}=x_{1} z_{1}=z_{1} y_{1} .
\end{aligned}
$$

Indeed,

$$
\begin{aligned}
y_{1} w_{1} & =w_{1} y w_{1}=y w_{1} \geq(x y) w_{1}=(x y)((x y)(z y) w) \\
& =(x y)(x(z y) w)=(x y)((x y)(z y w))= \\
& =(x y)(z y w)=x(z y) w=(x y)(z y) w=w_{1}, \\
x_{1} w_{1} & =(z y)(x y) y_{1} w_{1} \geq y_{1} w_{1}=w_{1}, \\
z_{1} w_{1} & =(x y)(z y) y_{1} w_{1} \geq y_{1} w_{1}=w_{1}
\end{aligned}
$$

and the other ones are consequences of $\Gamma_{0}$.

Thus $\left\{x_{1}, y_{1}, z_{1}, w_{1}\right\}$ constitutes an order algebra isomorphic to $\mathbf{D}$, unless some of these elements are equal. All equalities imply that $x_{1}=z_{1}$.

So suppose that $x_{1}=z_{1}$; i.e., $\mathcal{V}$ satisfies the equation

$$
(z y)(x y)((x y)(z y) w y)=(x y)(z y)((x y)(z y) w y) .
$$

In this equation, replace $y$ by $v, w$ by $u$, and then replace $x$ by $x y$ and $z$ by $z y$. This yields

$$
(z y v)(x y v)((x y v)(z y v) u v)=(x y v)(z y v)((x y v)(z y v) u v) .
$$

Using (e1) and three-variable equations, the left side equals

$$
(z y)(x y) v((x y)(z y) v u v)=(z y)(x y)((x y)(z y) u v) .
$$

Of course, the right side equals

$$
(x y)(z y)((x y)(z y) u v)=(x y)(z y)(u v) .
$$

So we see that if $x_{1}=z_{1}$ then $\mathcal{V}$ satisfies (e2), and hence $\mathcal{V} \subseteq \mathcal{P}_{3}$ by 4.9 .

Theorem 4.11. Let $\mathcal{V}$ be a variety satisfying the equations $\Gamma_{0}$ together with (e0). Then $\mathcal{V}$ satisfies (e1) if and only if $\mathbf{N} \notin \mathcal{V}$.

Proof. It is easy to check that $\mathbf{N}$ does not satisfy (e1). (Just evaluate $x=c$, $y=b, z=a$ and $u=d$, as represented in Fig. 3.) Now according to 4.3, it is sufficient to prove that $\mathcal{V}$ either contains $\mathbf{N}$ or satisfies (e3). We work in the free algebra $\mathbf{F}$ over $\{x, y, z, w\}$ in the variety defined by $\Gamma_{0}$ plus $(\mathrm{e} 0)$. Let $\varphi$ be the homomorphism of $\mathbf{F}$ onto $\mathbf{N}$ mapping $x \mapsto a, y \mapsto b, z \mapsto c, w \mapsto d$. 
We will first find a six-element subalgebra which maps onto $\mathbf{N}$ under $\varphi$. Define

$$
\begin{aligned}
& b=z(x y) w y, \\
& a=z(x y b), \\
& d=a(y(z(x y) w)) .
\end{aligned}
$$

There should result no confusion from using the same symbols for these elements of $\mathbf{F}$ as for their images under $\varphi$.

Claim 1: $a<b, a \| d$ (i.e., $a d=d$ and $d a=a$ ), and $b \| d$. Moreover, $z a=a$.

In proving this, note first that it is obvious that $a<b$ and $a d=d$. We see as follows that $d a=a$ :

$$
\begin{aligned}
d a & =(y(z(x y) w)) a=(y(z(x y) w)) a y \\
& =z(x y) w a y=z(x y) w y a y \\
& =z(x y) w y a=b a=a .
\end{aligned}
$$

Next,

$$
\begin{aligned}
b d & =(z(x y) w) y \cdot a(y(z(x y) w))=q y \cdot a(y q) \\
& =q y \cdot a(q y \cdot y q)=a \cdot y q=d .
\end{aligned}
$$

Next,

$$
\begin{aligned}
a & =z(x y b)=z(z(x y) b) \\
& =z(z(x y)(z(x y) w y))=z(z(x y)(w y)) \\
& =z((x y)(w y))=z(x(w y)) .
\end{aligned}
$$

Finally, let $u=z(x y) w$. Then

$$
\begin{aligned}
d b & =a(y u)(u y)=a y(y u)(u y)=a y u(u y) \\
& =a y u y=a u y=z(x(w y)) u y \\
& =u y z((x y)(w y)) u y=u y z(u y z(x y)(w y)) u y \\
& =u y z(u y z(z(x y))(w y)) u y \\
& =u y z(u y z(z(x y))(z(x y)(w y))) u y \\
& =u y z(u y z(z(x y))(z(x y)(z(x y) w y))) u y \\
& =u y z(u y z(z(x y))(z(x y)(u y))) u y \\
& =u y z(u y z(z(x y))(u y)) u y \\
& =u y z(z(x y)(u y)) u y \\
& =z(z(x y)(u y)) u y=z((x y)(u y)) u y \\
& =(z y)((x y)(u y)) u y=(z y)(x y)(u y) u y \\
& =z(x y)(u y) u w y=z(x y) w(u y) u w y \\
& =z(x y) w(u y) u y=u(u y) u y=u y u y=u y=b .
\end{aligned}
$$

It follows that $\{a, b, d\}$ is a subalgebra of $\mathbf{F}$. Moreover, since $b a=d a=$ $z a=a$, we have $p a=a$ for every $p$ in the subalgebra generated by $\{a, b, d, z\}$. 
We now consider the subalgebra $\mathbf{S}$ generated by $\{a, b, d, z b d\}$. We have $p a=a$ for all $p \in \mathbf{S}$. And it is easy to see that, for $H=S \cap \varphi^{-1}(c)$, we have $S=H \cup\{a, b, d\}$.

Claim 2: $H$ is identical with the set

$$
H^{\prime}=\{z b d, z d b, a \cdot z d b\}
$$

To prove it, we must show that

$$
H^{\prime} d \cup H^{\prime} b \subseteq H^{\prime}
$$

and that

$$
a H^{\prime} \cup b H^{\prime} \cup d H^{\prime} \cup H^{\prime} H^{\prime} \subseteq H^{\prime} .
$$

Here is a sample of the calculations to show that $H^{\prime}$ is closed under multiplying on the left by $a, b, d$ and on the right by $d, b$.

We have

$$
a(z d b) d=a d b(z d b) d=b(z d b)=z d b
$$

and

$$
d(a(z d b))=d b(a(z d b))=b(a(z d b))=a(z d b) .
$$

Other calculations are very similar.

The proof that $H^{\prime}$ is closed under multiplication is very easy. We have

$$
z d b(z b d)=z d b d(z b d)=z b d(z b d)=z b d
$$

and analogously, $z b d(z d b)=z d b$. Obviously, $a(z d b)(z d b)=a(z d b)$ and $z d b(a(z d b))=a(z d b)$. We have

$$
a(z d b)(z b d)=a d(z d b)(z b d)=d(z d b)(z b d)=(z d b)(z b d)
$$

and

$$
z b d(a(z d b))=z d b d(a(z d b))=z d b(a(z d b))=a(z d b) .
$$

So $S=H^{\prime} \cup\{a, b, d\}$ is the universe of a subalgebra of $\mathbf{F}$.

If $\mathcal{V}$ does not contain $\mathbf{N}$, then the kernel of the map of $\mathbf{S}$ into the free algebra in $\mathcal{V}$ generated by $x, y, z, w$ must not be contained in the kernel of $\varphi$ restricted to $S$. This means that $\mathcal{V}$ must identify two members of $S$ not both of which are in $H^{\prime}$. Considering the way the operation works in the factor of $\mathbf{S}$ through the kernel of $\varphi$ (restricted to $S$ ), we see that $\mathcal{V}$ satisfies some equation $b=\gamma$ where $\gamma \in H^{\prime}$. Now $b=z b d$ implies $b=z b d b=z d b$. Also, $b=a(z d b)$ implies $b=b d b=a(z d b) d b=z d b d b=z d b$. 
Thus we have the equation $b=z d b$. We shall show that this is equivalent to (e3). Recall that $a=z(x(w y))$ and $d=a(y(z(x y) w))$. We calculate

$$
\begin{aligned}
z d & =z(z(x(w y))(y(z(x y) w))) \\
& =z(x(w y)(y(z(x y) w))) \\
& =z(x(w y) y(y(z(x y) w))) \\
& =z(x(w y) y(z(x y) w)) \\
& =z(x(w y)(z(x y) w)) \\
& =z(x(w y)(z(x y) w) w) \\
& =z(x w(w y)(z(x y) w) w) \\
& =z(x w y(z(x y) w) w) \\
& =z(x y(z(x y) w) w) \\
& =z(z(x y)(z(x y) w)) \\
& =z(z(x y) w) \\
& =z(x y w)
\end{aligned}
$$

And then

$$
\begin{aligned}
z d b & =z(x y w)(z(x y) w y) \\
& =z(z(x y) w)(z(x y) w y) \\
& =z(z(x y) w) y \\
& =z(x y w) y .
\end{aligned}
$$

Thus the equation $b=z d b$ is equivalent to $z(x y) w y=z(x y w) y$, or, changing variables, to $x(y z) w z=x(y z w) z$, which is the equation (e3).

Theorem 4.12. The equations of $\Gamma_{0}$, together with the equation (e1), are a base for the equational theory of $\mathbf{D}$.

Proof. We have to prove that every equation which is satisfied in $\mathbf{D}$, belongs to $\Gamma_{2}$. If two terms $w, w^{\prime}$ are $\Gamma_{2}$-equivalent, we will also say that $w$ is reducible to $w^{\prime}$. We already know that every term is reducible to an $x$ normal term $w^{\prime}$, for some variable $x$. (This has been proved in 3.5.) We can use the associative law at will within an $x$-normal term, treating the constituents as indecomposable variables.

If $\mathbf{D}$ satisfies $w=w^{\prime}$ where $w, w^{\prime}$ are $x$-normal, $y$-normal, respectively, then $x$ and $y$ are the same variable, and the terms contain the same variables, and in fact, the dominant (rightmost) constituents in $w, w^{\prime}$ are equal. Our task is to show that assuming $\mathbf{D} \models w=w^{\prime}$ with $w, w^{\prime}$ both $x$-normal, then $w$ is reducible to $w w^{\prime}$ (which is $x$-normal) and $w^{\prime}$ is reducible to $w^{\prime} w$.

This reduces to: If $w(a x), w^{\prime}$ are $x$-normal in the same variables with $a x$ the dominant constituent of $w(a x)$ and if $\mathbf{D}=w(a x) w^{\prime}=w w^{\prime}$ then $w(a x) w^{\prime}$ is $\Gamma_{2}$-equivalent to $w w^{\prime}$. 
Now if $\mathbf{D}=w(a x) w^{\prime}=w w^{\prime}$ then where $a=y_{1} \cdots y_{n}$ and $b=y_{i} \cdots y_{n}$, since $a x=a x(b x)$ and $a x w^{\prime}=a x w^{\prime}(b x) w^{\prime}$ hold in $\mathcal{P}^{3}$, we have that in $\mathbf{D}$,

$$
w w^{\prime}=w w^{\prime}(a x) w^{\prime}=w w^{\prime}(b x) w^{\prime}=w(b x) w^{\prime} .
$$

This means that our task reduces to the following:

(D1) Suppose that $w, w^{\prime}$ are $x$-normal with the same variables and $c x$ is left associated without repeats, $z$ is a variable, $\operatorname{var}(c) \cup\{z\} \subseteq \operatorname{var}(w)$, and $c^{\prime}=z y_{i} \cdots y_{n}$ where $c=y_{i} \cdots y_{n}$. Then if $\mathbf{D} \models w(c x) w^{\prime}=w\left(c^{\prime} x\right) w^{\prime}$, the two terms are $\Gamma_{2}$-equivalent.

Claim 1: Suppose that $w, w^{\prime}, c x, z, c^{\prime} x$ are as above and $\mathbf{D} \models w(c x) w^{\prime}=$ $w\left(c^{\prime} x\right) w^{\prime}$. Then either (i) $w$ has a constituent $a x=u_{1} \cdots u_{a} z v_{1} \cdots v_{b} x$ such that $\left\{v_{1}, \ldots, v_{b}\right\} \subseteq \operatorname{var}(c)$; or else (ii) $w^{\prime}$ has a constituent $a x$ with this property. Moreover if (ii) fails and we write $w^{\prime}=w^{\prime \prime}(a x) w^{\prime \prime \prime}$ where we have exposed the rightmost such constituent $a x$ satisfying (i), then $\mathbf{D} \models$ $(a x) w^{\prime \prime \prime}(c x) w^{\prime}=(a x) w^{\prime \prime \prime}\left(c^{\prime} x\right) w^{\prime}$.

To prove the first subclaim, suppose that (i) and (ii) fail. Then evaluate the variables in $\mathbf{D}$ by mapping $z$ to $a, x$ to $b$, all variables of $c$ to $b$, and all remaining variables to $d$. Then $w(c x) w^{\prime}$ will evaluate as $b$, while $w\left(c^{\prime} x\right) w^{\prime}$ evaluates as $a$. So either (i) or (ii) holds. Now suppose that (ii) fails, (i) holds, and $w=w^{\prime \prime}(a x) w^{\prime \prime \prime}$ as indicated in the second subclaim. Suppose that we have an evaluation $p$ of variables in $\mathbf{D}$ under which $\lambda=a x w^{\prime \prime \prime}(c x) w^{\prime}$ and $\gamma=a x w^{\prime \prime \prime}\left(c^{\prime} x\right) w^{\prime}$ return different values. This means, among other things, that $p(x)=b$ and $p\left(w^{\prime}\right)=b$ and $p(c) \neq p\left(c^{\prime}\right)$. Then $p(u)=b$ for all variables $u \in c$, else $p(c)=p\left(c^{\prime}\right)$. Thus $p(c x)=b$ and $p\left(c^{\prime} x\right) \in\{a, c, d\}$ and so $p(z) \neq d$ and we get $p(z)=p\left(c^{\prime} x\right) \in\{a, c\}$. Then $p\left(c^{\prime} x w^{\prime}\right)=$ $p(z)=p\left(a x w^{\prime \prime \prime}\left(c^{\prime} x\right) w^{\prime}\right)=p\left(w\left(c^{\prime} x\right) w^{\prime}\right)$ since the elements $a, c$ are right zeros in D. Since $p(\lambda) \neq p(\gamma)$ while $p\left(w(c x) w^{\prime}\right)=p\left(w\left(c^{\prime} x\right) w^{\prime}\right)$, we have that $p(\lambda) \neq p\left(w^{\prime \prime}\right) p(\lambda)$. Thus $p(\lambda)$ is not a right zero, so it can only be $b$. But then $p\left(w^{\prime \prime \prime}(c x) w^{\prime}\right)=b$. However, we have $p(a x)=p(z)$ since the variables to the right of $z$ in $a$ are all getting the value $b$. This implies $p(\lambda)=p(z) b=$ $p(z) \neq b$. The contradiction proves the claim.

In case (ii) of the claim, we easily see that $w(c x) w^{\prime}$ is $\Gamma_{2}$-equivalent to $w\left(c^{\prime} x\right) w^{\prime}$, as follows. We have $w^{\prime}=a x w^{\prime}$. Thus (where $=$ denotes $\Gamma_{2}$-reducibility, $c=y_{1} \cdots y_{n}$ as above, $a=u_{1} \cdots u_{a} z v_{1} \cdots v_{b}$, and $c^{\prime \prime}=$ $\left.a y_{1} \cdots y_{n}\right)$

$$
\begin{aligned}
w(c x) w^{\prime} & =w((c x)(a x)) w^{\prime} \\
& =w\left(c^{\prime \prime} x\right)(a x) w^{\prime} \\
& =w\left(u_{1} \cdots u_{a} z y_{1} \cdots y_{n} x\right)(a x) w^{\prime} \\
& =w\left(a x z y_{1} \cdots y_{n} x\right)(a x) w^{\prime} \\
& =w\left(c^{\prime} x\right)(a x) w^{\prime} \\
& =w\left(c^{\prime} x\right) w^{\prime}
\end{aligned}
$$


Thus we are reduced to proving the special case of (D1) where

$$
w=u_{1} \cdots u_{a} z v_{1} \cdots v_{b} x w^{\prime \prime \prime}
$$

with $\left\{v_{1}, \ldots, v_{n}\right\} \subseteq \operatorname{var}(c)$, and we have exposed the leftmost constituent of $w$. It is easy to see that in this case,

$$
\mathbf{D} \models z v_{1} \cdots v_{b} x w^{\prime \prime \prime}(c x) w^{\prime}=z v_{1} \cdots v_{b} x w^{\prime \prime \prime}\left(c^{\prime} x\right) w^{\prime} .
$$

Thus we are reduced to proving equations of this sort. Moreover, when $\mathbf{D}$ satisfies such an equation, as above, then where $w^{\prime \prime \prime}=a_{1} x\left(a_{2} x\right) \cdots\left(a_{m} x\right)$ in $x$-normal form and $a x$ denotes $z v_{1} \cdots v_{b} x$, we have that the terms

$$
\begin{aligned}
& a x w^{\prime \prime \prime}(c x) w^{\prime}, \\
& a x(c x)\left(a_{1} x\right)(c x) \cdots\left(a_{m} x\right)(c x) w^{\prime}, \\
& a x\left(c^{\prime} x\right)\left(a_{1} x\right)(c x) \cdots\left(a_{m} x\right)(c x) w^{\prime}
\end{aligned}
$$

are $\mathbf{D}$-equivalent; the terms

$$
\begin{aligned}
& c^{\prime} x\left(a_{1} x\right)(c x) \cdots\left(a_{m} x\right)(c x) w^{\prime}, \\
& c^{\prime} x\left(a_{1} x\right)\left(c^{\prime} x\right)\left(a_{2} x\right)(c x) \cdots\left(a_{m} x\right)(c x) w^{\prime}
\end{aligned}
$$

are $\mathbf{D}$-equivalent; the terms

$$
\begin{aligned}
& c^{\prime} x\left(a_{2} x\right)(c x)\left(a_{2} x\right)(c x) \cdots\left(a_{m} x\right)(c x) w^{\prime}, \\
& c^{\prime} x\left(a_{2} x\right)\left(c^{\prime} x\right)\left(a_{3} x\right)(c x) \cdots\left(a_{m} x\right)(c x) w^{\prime}
\end{aligned}
$$

are $\mathbf{D}$-equivalent; and so on, up to the $\mathbf{D}$-equivalence of $c^{\prime} x\left(a_{m} x\right)(c x) w^{\prime}$ and $c^{\prime} x\left(a_{m} x\right)\left(c^{\prime} x\right) w^{\prime}$. If we can prove that all of these are $\Gamma_{2}$-equivalences, then we can conclude that $a x w^{\prime \prime \prime}(c x) w^{\prime}$ is $\Gamma_{2}$-equivalent to

$$
a x\left(c^{\prime} x\right)\left(a_{1} x\right)\left(c^{\prime} x\right) \cdots\left(a_{m} x\right)\left(c^{\prime} x\right) w^{\prime}
$$

and thence to $a x w^{\prime \prime \prime}\left(c^{\prime} x\right) w^{\prime}$, as desired.

So our task reduces to

(D2) Show the $\Gamma_{2}$-equivalence of terms $a x\left(a^{\prime} x\right)(c x) w^{\prime}$ and $a x\left(a^{\prime} x\right)\left(c^{\prime} x\right) w^{\prime}$ where their equality holds in $\mathbf{D}$, and where $a^{\prime} x w^{\prime}$ is an $x$-normal form which contains all the variables of $a^{\prime}$ and $c^{\prime}$, and $a=z v_{1} \cdots v_{m}, c=y_{1} \cdots y_{n}$, $c^{\prime}=z y_{1} \cdots y_{n}$ and $\left\{v_{1}, \ldots, v_{m}\right\} \subseteq\left\{y_{1}, \ldots, y_{n}\right\}$.

Now, in this situation, we can write $a^{\prime}=q z u_{1} \cdots u_{k}$ and then the equations $a x\left(a^{\prime} x\right)(c x) w^{\prime}=a x\left(a^{\prime} x\right)\left(z u_{1} \cdots u_{k} y_{1} \cdots y_{n} x\right) w^{\prime}$ and $a x\left(a^{\prime} x\right)\left(c^{\prime} x\right) w^{\prime}=$ $a x\left(a^{\prime} x\right)\left(u_{1} \cdots u_{k} z y_{1} \cdots y_{n} x\right) w^{\prime}$ are provable in $\mathcal{P}^{3}$; and each of the equations

$$
\begin{gathered}
\\
a x\left(a^{\prime} x\right)\left(u_{1} \cdots u_{i} z u_{i+1} \cdots u_{k} y_{1} \cdots y_{n} x\right) w^{\prime}= \\
=a x\left(a^{\prime} x\right)\left(u_{1} \cdots u_{i} u_{i+1} z u_{i+2} \cdots u_{k} y_{1} \cdots y_{n} x\right) w^{\prime}
\end{gathered}
$$

with $i=0, \ldots k-1$ is true in $\mathbf{D}$. Hence our task reduces to proving these equations. This is formulated as 
(D3) To show the $\Gamma_{2}$-equivalence of terms

$$
\begin{aligned}
& z v_{1} \ldots v_{m} x\left(\text { puu }_{1} \cdots u_{k} x\right)\left(q z u y_{1} \cdots y_{n} x\right) w \\
& z v_{1} \ldots v_{m} x\left(\text { puu }_{1} \cdots u_{k} x\right)\left(q u z y_{1} \cdots y_{n} x\right) w
\end{aligned}
$$

when they are D-equivalent, and $\left\{v_{1}, \ldots, v_{m}, u_{1}, \ldots, u_{k}\right\} \subseteq\left\{y_{1}, \ldots, y_{n}\right\}$. Here, $w$ is an $x$-normal term containing all the variables appearing in these terms, and all expressions $(\cdots x)$ are left-associated without repeating variables.

Now in the situation of (D3), assuming the equality holds in $\mathbf{D}$, there must be some constituent

$$
a x=t_{1} \cdots t_{i-1} u t_{i} \cdots t_{j-1} z t_{j} \cdots t_{k} x
$$

or

$$
a x=t_{1} \cdots t_{i-1} z t_{i} \cdots t_{j-1} u t_{j} \cdots t_{k} x
$$

of $w$ such that $\left\{t_{j}, \ldots, t_{k}\right\} \subseteq\left\{y_{1}, \ldots, y_{n}\right\}$ - else by evaluating in $\mathbf{D}$ with $x=b=y_{1}, \ldots, y_{n}, u=a, z=c$, and all other variables equal to $d$, we show that $\mathbf{D}$ does not satisfy the equation. Just as above, this reduces us to

(D4) Show the $\Gamma_{2}$-equivalence of terms

$$
\begin{aligned}
& z v_{1} \ldots v_{m} x\left(\text { puu }_{1} \cdots u_{k} x\right)\left(q z u y_{1} \cdots y_{n} x\right)\left(r t_{1} \cdots t_{\ell} x\right) \\
& z v_{1} \ldots v_{m} x\left(\text { puu }_{1} \cdots u_{k} x\right)\left(q u z y_{1} \cdots y_{n} x\right)\left(r t_{1} \cdots t_{\ell} x\right)
\end{aligned}
$$

where $r=u$ or $r=z$ and where $\left\{v_{1}, \ldots, v_{m}, u_{1}, \ldots, u_{k}, t_{1}, \ldots, t_{\ell}\right\} \subseteq\left\{y_{1}, \ldots, y_{n}\right\}$. Note that the equality of these terms is an identity of $\mathbf{D}$. Also, if $r=z$, we can omit the initial $z v_{1} \cdots v_{m} x$ and the equation still holds in $\mathbf{D}$.

Here is how we conclude the proof. Consider the case $r=u$ in (D4). Write the equation as

$$
\alpha \cdot \beta \cdot \gamma \cdot \delta=\alpha \cdot \beta \cdot \gamma^{\prime} \cdot \delta .
$$

Transform the left-side via

$$
\begin{aligned}
L S & =(\alpha \beta)\left((q z u) x y_{1} \cdots y_{n} x\right) \delta \\
& =(\alpha \beta)\left(\left([\alpha \beta(q z u x)] y_{1} \cdots y_{n} x\right) \delta\right) \\
& =(\alpha \beta)\left([\alpha x(\beta x)(q z u x)(\delta x)] y_{1} \cdots y_{n} x\right) \delta \\
& =(\alpha \beta)\left(\left[\alpha y_{1} \cdots y_{n} x\right]\left[\beta y_{1} \cdots y_{n} x\right] \gamma\left[\delta y_{1} \cdots y_{n} x\right]\right) \delta
\end{aligned}
$$

This is by equation (e1). Now $\alpha y_{1} \cdots y_{n} x$ reduces to $z x y_{1} \cdots y_{n} x, \beta y_{1} \cdots y_{n} x$ reduces to $\operatorname{pux}_{1} \cdots y_{n} x, \delta y_{1} \cdots y_{n} x$ reduces to $u x y_{1} \cdots y_{n} x$. We make these transformations and then pull $y_{1}, \cdots y_{n}$ back out to get

$$
=(\alpha \beta)\left((z x)(p u x)(q z u x)(u x) y_{1} \cdots y_{n} x\right) \delta .
$$

Now it suffices to transform $z x(p u x)(q z u x)(u x)$ into $z x(p u x)(q u z x)(u x)$ and then undo all the above transformations, keeping (quzx) intact, in order to arrive at $\alpha \cdot \beta \cdot \gamma^{\prime} \cdot \delta$, showing that the two terms are $\Gamma_{2}$-equivalent. But the equation

$$
z x(p u x)(q z u x)(u x)=z x(p u x)(q u z x)(u x)
$$


is a variant of (e6).

The other possibility, $r=z$, yields to the same process, using ultimately

$$
(p u x)(q z u x)(x z)=(p u x)(q u z x)(z x)
$$

which is a variant of (e5).

Since (e5) and (e6) belong to $\Gamma_{2}$ (by 4.4 and 4.6), we are done.

Theorem 4.13. The varieties $\mathcal{P}_{\mathbf{L}}, \mathcal{P}_{\mathbf{N}}$ and $\mathcal{P}_{\mathbf{D}}$ are three different covers of the variety $\mathcal{P}_{3}$. Every subvariety of $\mathcal{P}$ properly containing $\mathcal{P}_{3}$ contains one of these three covers. Also, the equations of $\Gamma_{0}$ together with $(e 2)$ are a base for the equational theory of $\mathcal{P}_{3}$.

Proof. Where $a=x z \cdot y z, b=y z \cdot x z, c=(x \cdot y z)(x \cdot w z)$ and $d=(x \cdot w z)(x \cdot y z)$, consider the equations

$(\mathrm{e} 2.1) \quad(a \cdot b u v)(b \cdot u v)=(b \cdot u v)(a \cdot b u v)$,

$(\mathrm{e} 2.2) \quad d(c x \cdot d x z)=c(d x z)$.

It is easy to verify that

$$
\begin{array}{lllll}
\mathbf{L} \not \models(e 0) & \mathbf{L} \not \models(e 1) & \mathbf{L} \not=(e 2) & \mathbf{L} \models(e 2.1) & \mathbf{L}=(e 2.2) \\
\mathbf{N}=(e 0) & \mathbf{N} \not=(e 1) & \mathbf{N} \not=(e 2) & \mathbf{N} \not \models(e 2.1) & \mathbf{N}=(e 2.2) \\
\mathbf{D}=(e 0) & \mathbf{D}=(e 1) & \mathbf{D} \not=(e 2) & \mathbf{D} \not \models(e 2.1) & \mathbf{D} \not \models(e 2.2)
\end{array}
$$

Clearly, $\mathcal{P}_{\mathbf{L}}, \mathcal{P}_{\mathbf{N}}, \mathcal{P}_{\mathbf{D}}$ contain $\mathcal{P}_{3}$ and since they do not satisfy (e2), by 4.9 they properly contain $\mathcal{P}_{3}$. It follows from above that these three varieties are pairwise incomparable. Then it follows from 4.1, 4.10 and 4.11 that every subvariety of $\mathcal{P}$ properly containing $\mathcal{P}_{3}$ contains at least one of them. The last statement follows from 4.9 and the fact that these three covers do not satisfy (e2).

\section{Simple Order Algebras}

Theorem 5.1. Every finite order algebra with $n$ elements $(n \geq 4)$ can be embedded into a simple order algebra with $2 n+1$ elements.

Proof. Let $\mathbf{A}$ be an order algebra with elements $a_{0}, \ldots, a_{n-1}$. We can suppose that $a_{i} \leq a_{j}$ implies $i \leq j$. Let $\mathbf{F}$ be the fence with elements $b_{0}, \ldots, b_{n}$ and coverings $b_{0}<b_{n}>b_{1}<b_{n-1}>b_{2}<\cdots$. Define an order algebra on $B=A \cup F$ in such a way that both $\mathbf{A}$ and $\mathbf{F}$ are subalgebras, $a_{i} \not \leq b_{j}$ for all $i, j$, and $b_{i} \leq a_{j}$ if and only if $i \leq j$. One can easily prove that $\mathbf{B}$ is simple.

\section{Free Spectra}

For a variety $\mathcal{V}$ we let $f_{\mathcal{V}}(n)=\left|\mathbf{F}_{\mathcal{V}}(n)\right|$ be the cardinality of the free algebra on $n$ generators. The function $f_{\mathcal{V}}$ is called the free spectrum of $\mathcal{V}$. In this section we will show that $f_{\mathcal{P}}(n)$ has an upper and lower bound both of the form

$$
2^{2^{\frac{n^{2}}{4}+o\left(n^{2}\right)}}
$$


We also prove the same result for the variety generated by tournaments except $n^{2} / 4$ is replaced with $n^{2} / 2$.

Herein all logarithms are base 2 .

Theorem 6.1. The free spectrum of $\mathcal{P}$ satisfies

$$
2^{2^{\frac{n^{2}}{4}-\frac{5 n}{2}-2 \log n}} \leq f_{\mathcal{P}}(n) \leq 2^{2^{\frac{n^{2}}{4}+n \log n+3 n / 2+o(n)}}
$$

for large numbers $n$, where $o(n)$ denotes a function $g(n)$ such that $\lim _{n \rightarrow \infty} g(n) / n=0$.

Proof. We begin with the upper bound. Since an $n$-generated order algebra has at most $n$ elements, $\mathbf{F}_{\mathcal{P}}(n)$ can be represented as a subdirect product of the free algebras $\mathbf{F}_{\boldsymbol{V}(\mathbf{A})}(n)$, taken over nonisomorphic order algebras $\mathbf{A}$ with at most $n$ elements. Each of these free algebras satisfies $\left|\mathbf{F}_{\boldsymbol{V}(\mathbf{A})}(n)\right| \leq$ $n^{n^{n}}=2^{2^{n \log n+\log \log n}}$. Thus

$$
\left|\mathbf{F}_{\mathcal{P}}(n)\right| \leq\left(2^{2^{n \log n+\log \log n}}\right)^{N}
$$

where $N$ is the number of isomorphism classes of ordered sets with at most $n$ elements.

By the theorem of Kleitman and Rothschild [8] there are at most

$$
2^{n^{2} / 4+3 n / 2+b \log n}
$$

ordered sets on an $n$ element set, for some constant $b$. Multiplying this by $n$ only has the effect of increasing $b$ by 1 so $N \leq 2^{n^{2} / 4+3 n / 2+c \log n}$ with $c=b+1$. Thus (for a slightly larger $c$ )

$$
\left|\mathbf{F}_{\mathcal{P}}(n)\right| \leq\left(2^{2^{n \log n+\log \log n}}\right)^{N} \leq 2^{2^{n^{2} / 4+3 n / 2+n \log n+c \log n}}
$$

proving the upper bound.

For the lower bound let $n=k+5$ where $k=2 m$ is even and let $X=$ $\left\{x_{0}, \ldots, x_{k-1}\right\} \cup\{y, z, u, v, w\}$. For each subset $S$ of $m \times m$ we can order $X$ by putting $w>v<u>z<y, y>x_{i}$ for $i<m$, and $x_{i}<x_{m+j}$ if $(i, j) \in S$. We let $\mathbf{A}_{S}$ be the corresponding order algebra.

Define terms $\tau_{i j}=z\left(x_{i} x_{m+j} y\right) u$. When evaluated in $\mathbf{A}_{S}$ (with the variables mapping to themselves) $\tau_{i j}=u$ if $(i, j) \in S$ and $\tau_{i j}=z$ otherwise.

For $T$ a nonvoid subset of $m \times m$ put $\lambda_{T}=\prod_{(i, j) \in T} \tau_{i j}$. The product is the left associated product of these terms in some order. In $\mathbf{A}_{S}$ we have $\lambda_{T}=u$ if $T \subseteq S$ and is $z$ otherwise. Thus if we let $\lambda_{T}^{\prime}=v \lambda_{T} w$ then in $\mathbf{A}_{S}$, $\lambda_{T}^{\prime}=v$ if $T \subseteq S$ and is $w$ otherwise.

Let $\mathcal{A}$ be the largest size antichain in the lattice of subsets of $m \times m$. Note $|\mathcal{A}| \geq 2^{m^{2}} / m^{2}$. For every nonempty subset $\mathcal{B}$ of $\mathcal{A}$ put $\mu_{\mathcal{B}}=\prod_{T \in \mathcal{B}} \lambda_{T}^{\prime}$. Notice that if $S \in \mathcal{A}$ then $\mu_{\mathcal{B}}$ evaluates in $\mathbf{A}_{S}$ to $w$ if $S \notin \mathcal{B}$ and to $v$ otherwise. If $\mathcal{B}$ and $\mathcal{B}^{\prime}$ are distinct subsets of $\mathcal{A}$ choose $S$ in one but not the other; say $S \in \mathcal{B}^{\prime}-\mathcal{B}$. Then in $\mathbf{A}_{S}$ the term $\mu_{\mathcal{B}}$ evaluates to $w$ while the term $\mu_{\mathcal{B}^{\prime}}$ evaluates to $v$. Hence the terms $\mu_{\mathcal{B}}$ are distinct. Putting $\mu_{\emptyset}=y$ 
we obtain $2^{|\mathcal{A}|}$ distinct elements of $\mathbf{F}_{\mathcal{P}}(n)$. Using this it is easy to get the lower bound of the theorem.

When $n-5$ is odd we can write $n-5=2 m+1$. In this case we can use subsets of $m \times(m-1)$ and prove the same lower bound.

A tournament is a commutative, idempotent groupoid such that $x y \in$ $\{x, y\}$. Tournaments can also be described as groupoids in which every two element subset forms a subgroupoid isomorphic to the two element semilattice. For $a, b$ in a tournament we let $a<b$ denote the fact that $a b=b a=a$. The next theorem achieves bounds on the free spectrum of the variety $\mathcal{T}$ generated by tournaments similar to what we obtained for $\mathcal{P}$.

Theorem 6.2. The free spectrum of the variety $\mathcal{T}$ generated by tournaments satisfies

$$
2^{2^{\frac{n^{2}}{2}-n \log n-6 n}} \leq f_{\mathcal{T}}(n) \leq 2^{2^{\frac{n^{2}}{2}+n \log n+o(n)}}
$$

for large numbers $n$, where $o(n)$ denotes a function $g(n)$ such that $\lim _{n \rightarrow \infty} g(n) / n=0$.

Proof. The number of (labeled) tournaments on a set with $n$ elements is $2\left(\begin{array}{l}n \\ 2\end{array}\right)$ and so arguments as in the last theorem give the upper bound.

For the lower bound let $k$ be an integer, $r=\lceil\log k\rceil$ and let

$$
X=\left\{x_{0}, \ldots, x_{k-1}\right\} \cup\left\{y_{0}, \ldots, y_{r-1}\right\} \cup\{z, u, v, w\}
$$

Let $P=\{(i, j): 0 \leq i<j<k\}$. For $T \subseteq P$ define a tournament $\mathbf{B}_{T}$ on $\left\{x_{0}, \ldots, x_{k-1}\right\}$ by specifying, for $i<j, x_{i}<x_{j}$ if $(i, j) \in T$ and $x_{j}<x_{i}$ otherwise. Extend this to an algebra $\mathbf{A}_{T}$ on $X$ by specifying, for $i<k$ and $t<r$

$$
\begin{array}{lcrl}
z & <y_{t} & z & <v \\
z & <u & w & <z \\
v & <u & x_{i} & <z \\
v & <w & u & <x_{i}
\end{array}
$$

We write $i$ base 2 as $i=i_{0}+i_{1} 2+\cdots+i_{t} 2^{t}+\cdots$ and define

$$
x_{i} y_{t}= \begin{cases}x_{i} & \text { if } i_{t}=0 \\ y_{t} & \text { if } i_{t}=1\end{cases}
$$

For $0 \leq i<j<k$ define $\tau_{i j}=x_{i} x_{j} y_{t} z u$, where $t$ is the largest integer such that $i_{t} \neq j_{t}$, that is, the most significant bit where they differ. In $\mathbf{A}_{T}$, $\tau_{i j}$ evaluates to $u$ if $(i, j) \in T$ and to $z$ otherwise. For $S$ a nonvoid subset of $P$, put $\lambda_{S}=\prod_{(i, j) \in S} \tau_{i j}$ and put $\lambda_{S}^{\prime}=\lambda_{S} v w$. In $\mathbf{A}_{T}, \lambda_{S}^{\prime}=v$ if $S \subseteq T$ and is $w$ otherwise. Just as in the proof of Theorem 6.1 this can be used

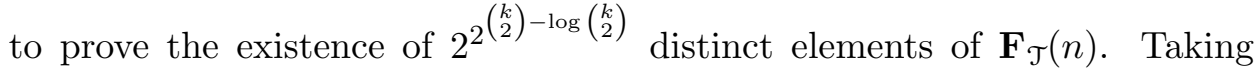
$k=n-\lceil\log n\rceil-4$, we have $k+\lceil\log k\rceil+4 \leq n$, and the lower bound given in the theorem follows easily. 
Of course the lower bounds of these theorems grow faster than the free spectrum of any finitely generated variety and so we obtain the next corollary which strengthens Corollary 2.2.

Corollary 6.3. Neither $\mathcal{P}$ nor $\mathcal{T}$ is a finitely generated variety. In fact neither variety is contained in a finitely generated variety.

The $G$-spectrum of a variety $\mathcal{V}$ is the function $g \mathcal{V}(n)$ which is the number of nonisomorphic $n$-generated algebras in $\mathcal{V}$.

Theorem 6.4. The G-spectra of $\mathcal{P}$ and $\mathcal{T}$ satisfy

$$
2^{2^{n^{2} / 4+o\left(n^{2}\right)}} \leq g_{\mathcal{P}}(n) \quad 2^{2^{n^{2} / 2+o\left(n^{2}\right)}} \leq g_{\mathcal{T}}(n)
$$

Proof. We give the proof for $\mathcal{P}$; the proof for $\mathcal{T}$ is similar. Let $\mathcal{A}$ be the antichain used in the proof of Theorem 6.1. For $\mathcal{B} \subseteq \mathcal{A}$ with $|\mathcal{B}| \geq 2$ let $\theta_{\mathcal{B}}$ be the congruence on $\mathbf{F}_{\mathcal{P}}(X)$ generated by identifying all $\lambda_{S}^{\prime}$, for $S \in \mathcal{B}$. If $S, T \in \mathcal{B}$ then, in $\mathbf{A}_{S}, \lambda_{S}^{\prime}=v$ while $\lambda_{T}^{\prime}=w$. Thus the natural map from $\mathbf{F}_{\mathcal{P}}(X)$ to $\mathbf{A}_{S}$ does not factor through $\mathbf{F}_{\mathcal{P}}(X) / \theta_{\mathcal{B}}$. On the other hand, if $S \notin \mathcal{B}$ then it does. Thus $\theta_{\mathcal{B}} \neq \theta_{\mathcal{B}^{\prime}}$ for distinct subsets of $\mathcal{A}$, each with at least two elements.

In $\mathbf{F}_{\mathcal{P}}(X)$ the elements of $X$ are irreducible in the strong sense that if $x=u v$ then $u=x$ and $v=x$. This can be seen by looking at the natural map from $\mathbf{F}_{\mathcal{P}}(X)$ onto the free semilattice over $X$. Since $\mathcal{T}$ also contains the variety of semilattices, $\mathbf{F}_{\mathcal{T}}(X)$ also has this property. Consequently if $x \in X$ then its $\theta_{\mathcal{B}}$ class contains only itself. Thus the image of $X$ in $\mathbf{F}_{\mathcal{P}}(X) / \theta_{\mathcal{B}}$ is precisely the set of irreducible elements. It follows that any isomorphism between $\mathbf{F}_{\mathcal{P}}(X) / \theta_{\mathcal{B}}$ and $\mathbf{F}_{\mathcal{P}}(X) / \theta_{\mathcal{B}^{\prime}}$ is induced by an automorphism of $\mathbf{F}_{\mathcal{P}}(X)$ which permutes $X$ and carries $\theta_{\mathcal{B}}$ onto $\theta_{\mathcal{B}^{\prime}}$. There are at most $n$ ! such automorphisms. Thus the number of non-isomorphic algebras among the algebras $\mathbf{F}_{\mathcal{P}}(X) / \theta_{\mathcal{B}}$ is at least the number of subsets of $\mathcal{A}$ with at least two elements, divided by $n$ !. The theorem follows from this.

\section{The Equational Theory of Order Algebras}

A term $t$ is said to be linear if every variable has at most one occurrence in $t$.

Theorem 7.1. Let $u, v$ be two linear terms such that the equation $(u, v)$ is satisfied in all order algebras. Then $u=v$.

Proof. Because the equation is satisfied in $\mathbf{O}_{\wedge}$, the two terms are equivalent with respect to the equational theory of semigroups. Let us proceed by induction on the length of $u$. If $u$ is a variable, there is nothing to prove. Let $u=u_{1} u_{2}$ and $v=v_{1} v_{2}$. If $u_{1}$ is shorter than $v_{1}$, consider the interpretation in $\mathbf{O}_{2+1}$, sending each variable in $u_{1}$ to $a$, the last variable of $u$ to $b$, and the remaining variables to $c$ (we have $a<b$, while $c$ is incomparable with both $a$ and $b$ ); under this interpretation, $u$ is evaluated to $a$, while $v$ is evaluated to $b$. If $u_{1}$ is longer than $v_{1}$, we obtain a contradiction symmetrically. Hence 


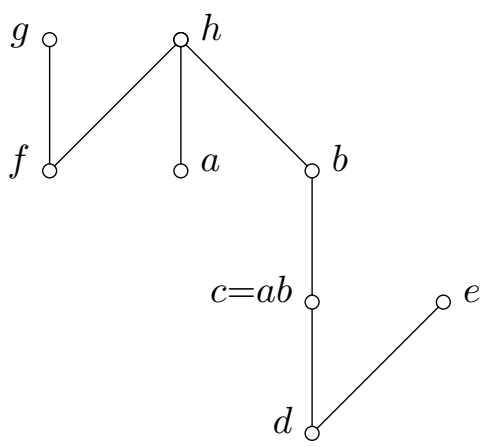

Fig. 4

$u_{1}$ is of the same length as $v_{1}$, and the two terms contain the same variables. Let $\mathbf{A}$ be an order algebra and let $f$ be an interpretation of the variables of $u_{1}$ in $\mathbf{A}$. Extend $\mathbf{A}$ to an order algebra $\mathbf{A}^{\prime}$ by adding a new largest element $e$, and extend $f$ to $f^{\prime}$ by sending all the variables of $u_{2}$ to $e$. Clearly, $u_{1}$ evaluates to the same element under $f$ as $u$ under $f^{\prime}$, and the same is true for $v_{1}$ and $v$. We conclude that the equation $\left(u_{1}, v_{1}\right)$ is satisfied in $\mathbf{A}$. By induction, $u_{1}=v_{1}$. We can prove $u_{2}=v_{2}$ in the same way, if we extend $\mathbf{A}$ to $\mathbf{A}^{\prime}$ by adding a new element incomparable to all the elements of $\mathbf{A}$.

Modulo the equational theory of order algebras, each term in at most three variables is equivalent to precisely one linear term. This is not true for terms in a larger number of variables: the free four-generated algebra in $\mathcal{P}$ has 1456 elements, while there are just 184 linear terms in four variables. (For more details see Example 8.7.)

We do not know whether there is an equational theory $E$ of groupoids with the property that every term is $E$-equivalent to precisely one linear term.

\section{EXAMPLES}

Example 8.1. Let $\mathbf{A}_{0}=\mathbf{P}_{0}[a b=c]$, where $\mathbf{P}_{0}=\{a, b, c, d, e, f, g, h\}$ is the order algebra with coverings $a<h, d<c<b<h, d<e, f<g, f<h$. (See Fig. 4.) Then $\mathbf{A}_{0} \notin \mathcal{P}$.

Proof. Suppose $\mathbf{A}_{0} \in \mathcal{P}$, so that $A_{0}=\varphi(S)$ for a homomorphism $\varphi$ of a subalgebra $\mathbf{S}$ of the product of finitely many finite order algebras $\mathbf{P}_{1}, \ldots, \mathbf{P}_{n}$. Take a minimal element $H$ in $\varphi^{-1}(h)$, a minimal element $E$ in $\varphi^{-1}(e)$, and a minimal element $G$ in $\varphi^{-1}(g)$. There exists a minimal element $A$ in $\varphi^{-1}(a)$ such that $A<H$ (if $A^{\prime}$ is any element of $\varphi^{-1}(a)$, take $A$ to be a minimal element of $\varphi^{-1}(a)$ such that $\left.A \leq A^{\prime} H\right)$. Similarly, choose minimal elements $B, D, F$ in $\varphi^{-1}(b), \varphi^{-1}(d), \varphi^{-1}(f)$ such that $B<H, D<A B$ and $F<G$; 
and put $C=A B$. It follows from the minimality of these elements that if $X, Y$ are the selected elements in $\varphi^{-1}(x), \varphi^{-1}(y)$ where $x, y$ are incomparable, then $X Y=Y$, unless either $(x, y)=(a, b)$ or $y=c$. For example, $H G=G$ and $E A=A$.

If $i \in\{1, \ldots, n\}$ is such that $A_{i} \leq B_{i}$, then $D_{i}=A_{i}$. In fact, we have $D A=A$, so that $D_{i} A_{i}=A_{i}$; but $D_{i} \leq A_{i} B_{i}=A_{i}$, so that $D_{i} A_{i}=D_{i}$.

Put

$$
\beta=(A \cdot F H) B, \quad \gamma=A(\beta(F \cdot D E H) G H) B .
$$

Clearly, $\varphi(\beta)=b$ and $\varphi(\gamma)=c$, so that $\beta \neq \gamma$. Easy computation shows that if $i \in\{1, \ldots, n\}$ is such that $A_{i} \leq B_{i}$ and $F_{i} \not \leq H_{i}$, then $\beta_{i}=\gamma_{i}=A_{i}$. For all other numbers $i \in\{1, \ldots, n\}$ one can verify that $\beta_{i}=\gamma_{i}=B_{i}$ (the verification should be divided into cases, corresponding to whether or not $\left.A_{i} \leq B_{i}, D_{i} \leq E_{i}, F_{i} \leq H_{i}\right)$. So, we get $\beta_{i}=\gamma_{i}$ for all $i$, contradicting $\beta \neq \gamma$.

Observe that the same proof shows $\mathbf{A}_{0} \notin \mathcal{P}$ if we add either $g<e$ or $f<e$ to the list of coverings in $\mathbf{A}_{0}$.

Theorem 8.2. Let $\mathbf{A} \in \mathcal{P}^{3}$ be a groupoid containing precisely one triple $a, b, c$ of elements such that $a b=c \notin\{a, b\}$. Suppose that both $a$ and $b$ are maximal in $\mathbf{A}$, and that $x<b$ implies $x \leq c$. Then $\mathbf{A} \in \mathcal{P}$.

Proof. Put $P=A-\{x: x \leq c\}$, and consider $\mathbf{P}$ as an order algebra with respect to the ordering $x \leq_{\mathbf{P}} y$ iff either $x \leq_{\mathbf{A}} y$ or $(x, y)=(a, b)$. Let $\mathbf{Q}$ be the order algebra on $A-\{c\}$. Denote by $S$ the set of all $(x, y) \in P \times Q$ satisfying the following four conditions:

$$
\begin{aligned}
& y=a \text { implies } x=a ; \\
& y=b \text { implies } x \in\{a, b\} ; \\
& x=a \text { implies either } y=a \text { or } y \leq b ; \\
& x=b \text { implies } y \leq b .
\end{aligned}
$$

Note that lemma 0.2 implies that for all $x \in A, x>c$ only if $x \geq b$, and $x<a$ only if $x<c$. Using this, it is not difficult to check that $\mathbf{S}$ is a subgroupoid of $\mathbf{P} \times \mathbf{Q}$. Define a mapping $\varphi$ of $\mathbf{S}$ into $\mathbf{A}$ by $\varphi(a, b)=c$ and $\varphi(x, y)=y$ in all other cases. It can be verified that $\varphi$ is a homomorphism of $\mathbf{S}$ onto $\mathbf{A}$.

An infinite fence is an example of an infinite simple algebra in $\mathcal{P}$. We do not know if such an example can be found among the algebras of $\mathcal{P}$ that are not order algebras. However, we are able at least to give an example of an infinite subdirectly irreducible algebra in $\mathcal{P}$ which is not an order algebra:

Example 8.3. Let $\mathbf{A}=\mathbf{P}[a b=c]$ where $\mathbf{P}$ is the order algebra with infinitely many elements $a, b, c, d, e, f, x_{i}, y_{i}(i=1,2, \ldots)$ and coverings $a<f$, $d<c<b<f, e<f, d<y_{1}, x_{i}<y_{i}$ and $x_{i}<y_{i+1}$. (See Fig. 5.) Then $\mathbf{A}$ is subdirectly irreducible and belongs to $\mathcal{P}$.

Proof. The monolith of $\mathbf{A}$ has only one non-singleton block $\{b, c\}$. Let $\mathbf{P}$ be the order algebra with elements $a, b, f, e, x_{i}, y_{i}$ and coverings $a<b<f$, 


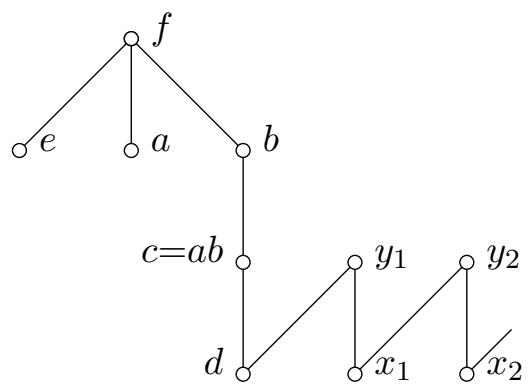

Fig. 5

$x_{i}<y_{i}, x_{i}<y_{i+1}$. Let $\mathbf{Q}$ be the order algebra with the same elements and one more covering $e<f$. Let $\mathbf{R}$ be the order subalgebra of $\mathbf{A}$ on $A-\{c\}$. Denote by $\mathbf{S}$ the subgroupoid of $\mathbf{P} \times \mathbf{Q} \times \mathbf{R}$ generated by the elements $(a, a, d)$ and $(x, x, x)$ where $x=a, b, e, f, x_{i}, y_{i}$. One can easily check that there is a homomorphism $\varphi$ of $\mathbf{S}$ onto $\mathbf{A}$, where

$$
\begin{aligned}
& \varphi^{-1}(a)=\{(a, a, a)\}, \\
& \varphi^{-1}(b)=\{(b, b, b),(a, b, b)\}, \\
& \varphi^{-1}(c)=\{(a, a, b)\}, \\
& \varphi^{-1}(d)=\{(a, a, d),(a, b, d),(b, b, d),((f, f, d),(a, e, d),(b, e, d),(c, e, d), \\
&\left.\left(y_{1}, y_{1}, d\right),\left(x_{1}, x_{1}, d\right)\right\} \\
& \varphi^{-1}(e)=\{(a, e, e),(b, e, e),(e, e, e),(f, e, e)\}, \\
& \varphi^{-1}(f)=\{(f, f, f)\}, \\
& \varphi^{-1}\left(x_{i}\right)=\left\{\left(x_{i}, x_{i}, x_{i}\right)\right\} \text { for } i \text { odd }, \\
& \varphi^{-1}\left(x_{i}\right)=\left\{\left(x_{i-1}, x_{i-1}, x_{i}\right),\left(y_{i}, y_{i}, x_{i}\right),\left(x_{i}, x_{i}, x_{i}\right),\left(y_{i+1}, y_{i+1}, x_{i}\right),\right. \\
&\left.\quad\left(x_{i+1}, x_{i+1}, x_{i}\right)\right\} \text { for } i \text { even, } \\
& \varphi^{-1}\left(y_{i}\right)=\left\{\left(y_{i}, y_{i}, y_{i}\right),\left(x_{i}, x_{i}, y_{i}\right)\right\} \text { for } i \text { odd, } \\
& \varphi^{-1}\left(y_{i}\right)=\left\{\left(y_{i}, y_{i}, y_{i}\right),\left(x_{i-1}, x_{i-1}, y_{i}\right)\right\} \text { for } i \text { even. }
\end{aligned}
$$

(This also gives us the list of elements in $\mathbf{S}$.)

Example 8.4. Similar constructions (we can do with the product of two order algebras) show that the subdirectly irreducible algebras $\mathbf{P}_{1}[a b=c]$, $\mathbf{P}_{2}[a b=c]$ and $\mathbf{P}_{3}[a b=c]$, where $\mathbf{P}_{1}, \mathbf{P}_{2}, \mathbf{P}_{3}$ are the three order algebras shown in Fig. 6, belong to $\mathcal{P}$.

Example 8.5. The five-element algebra in Fig. 7 does not belong to $\mathcal{P}$. It does not satisfy the equation $x w(x(z y)(w y))=x(z(w y))$ (evaluate $x=c$, $y=b, z=d$ and $w=a$ ), which is satisfied in all order algebras (the proof is not short, but can be done mechanicaally.)

Another proof is to suppose that the algebra is a homomorphic image, under a homomorphism $h$, of a subalgebra of a product of finitely many finite order algebras. Let $\alpha$ be a minimal element of $h^{-1}(a), \beta$ be a minimal 

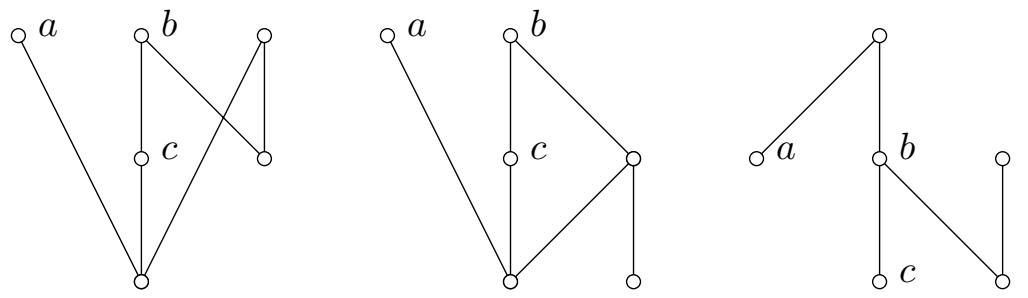

Fig. 6

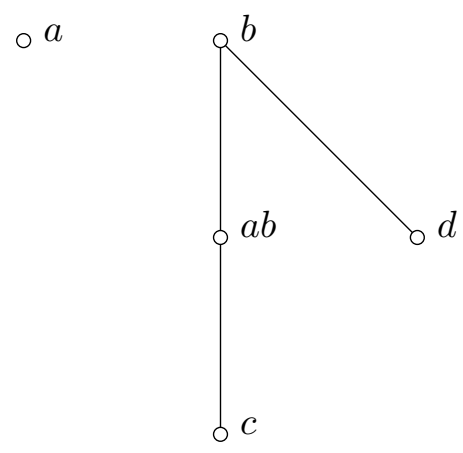

Fig. 7

element of $h^{-1}(b), \gamma$ be a minimal element of $h^{-1}(c)$ such that $\gamma<\alpha \beta$, and $\delta$ be a minimal element of $h^{-1}(d)$ such that $\delta<\beta$. The two distinct elements, $\gamma(\delta(\alpha \beta))$ and $\delta(\alpha \beta)$, are easily seen to be equal at each coordinate, which gives the contradiction.

By adding two elements to this algebra, one above $a$ and $b$ and the other above $c$ and $d$, we obtain a simple algebra belonging to $\mathcal{P}^{3}$ (but not to $\mathcal{P}$ ).

Example 8.6. It can be proved that there are just five five-element, subdirectly irreducible algebras in $\mathcal{P}^{3}$ that are not order algebras. They are the algebra from example 8.5, and the four algebras pictured in Fig. 8. These four algebras belong to $\mathcal{P}$. In fact, the first one generates the same variety as $\mathbf{O}_{L}$.

Example 8.7. We can precisely describe the free algebra in $\mathcal{P}$ over four generators $\{x, y, z, w\}$. The algebra contains 1456 elements. Sixty of them have $z$ as the last variable, $y$ as the next to the last variable, and $x$ as the next to the next to the last variable. Here is the list of these sixty elements:

$$
t_{1}=x y z \quad t_{2}=w x y z
$$



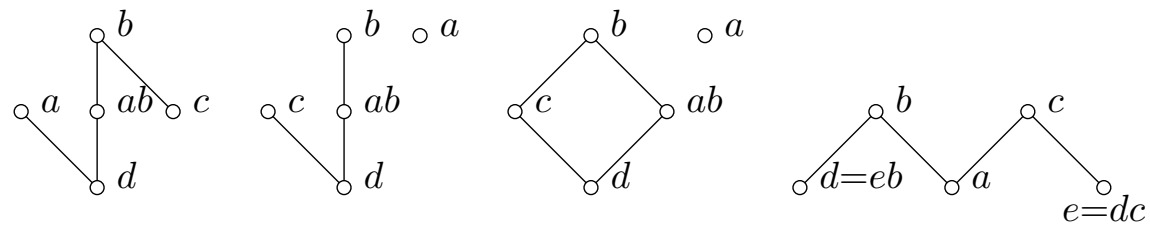

Fig. 8

$$
\begin{aligned}
& t_{3}=w(x z)(x y) z \\
& t_{5}=w(x z) y z \\
& t_{7}=w(x(y z))(x(z y)) z \\
& t_{9}=x(y z) \\
& t_{11}=w(x y)(x z)(y z) \\
& t_{13}=w y(x z)(y z) \\
& t_{15}=w(x(z y))(x(y z)) \\
& t_{17}=w(y x)(y z) \\
& t_{19}=w(x(z y))(w(y x) z)(y z) \\
& t_{21}=w(x y)(w(y x)(y z)) \\
& t_{23}=w y(w(y x)(y z)) \\
& t_{25}=w(x y)(w(y(z x)) z)(y z) \\
& t_{27}=w y(w(y(z x)) z)(y z) \\
& t_{29}=w(x(z y))(w(y(z x))(y z)) \\
& t_{31}=w x(y z) \\
& t_{33}=w(x(z y))(w x z)(y z) \\
& t_{35}=w(x z)(y z) \\
& t_{37}=w(x(z y))(w x(y z)) \\
& t_{39}=w(x(y z)) \\
& t_{41}=x(w(x y) z) \\
& t_{43}=w(y(z x))(w(x y) z) \\
& t_{45}=w(x(z y)) z \\
& t_{47}=w(y x)(w(x(z y)) z) \\
& t_{49}=w x(w(x(z y)) z) \\
& t_{51}=x(w(x z y) z) \\
& t_{53}=w(y(z x))(w(x z y) z) \\
& t_{55}=w(x(w(x y) z)) \\
& t_{57}=x(y w)(x y z) \\
& t_{59}=x w(x y z)
\end{aligned}
$$

$$
\begin{aligned}
& t_{4}=w(x z)(x(z y)) z \\
& t_{6}=w(x(y z))(x y) z \\
& t_{8}=w(x(y z)) y z \\
& t_{10}=w y x(y z) \\
& t_{12}=w(x(z y))(x z)(y z) \\
& t_{14}=w(x y)(x(y z)) \\
& t_{16}=w y(x(y z)) \\
& t_{18}=w(x y)(w(y x) z)(y z) \\
& t_{20}=w y(w(y x) z)(y z) \\
& t_{22}=w(x(z y))(w(y x)(y z)) \\
& t_{24}=w(y(z x)(y z) \\
& t_{26}=w(x(z y))(w(y(z x)) z)(y z) \\
& t_{28}=w(x y)(w(y(z x))(y z)) \\
& t_{30}=w y(w(y(z x))(y z)) \\
& t_{32}=w(x y)(w x z)(y z) \\
& t_{34}=w y(w x z)(y z) \\
& t_{36}=w(x y)(w x(y z)) \\
& t_{38}=w y(w x(y z)) \\
& t_{40}=w(x y) z \\
& t_{42}=w(y x)(w(x y) z) \\
& t_{44}=w x(w(x y) z) \\
& t_{46}=x(w(x(z y)) z) \\
& t_{48}=w(y(z x))(w(x(z y)) z) \\
& t_{50}=w(x z y) z \\
& t_{52}=w(y x)(w(x z y) z) \\
& t_{54}=w x(w(x z y) z) \\
& t_{56}=w(x y z) \\
& t_{58}=x(y(z w))(x y z) \\
& t_{60}=x(w(x y z)) \\
&
\end{aligned}
$$

They are precisely the elements below $x y z$. We have obtained them by running a computer program, which was designed so that the resulting terms are not equivalent to any shorter terms. The less-than relations between these terms are shown in Fig. 9. All the other elements of the free algebra, 


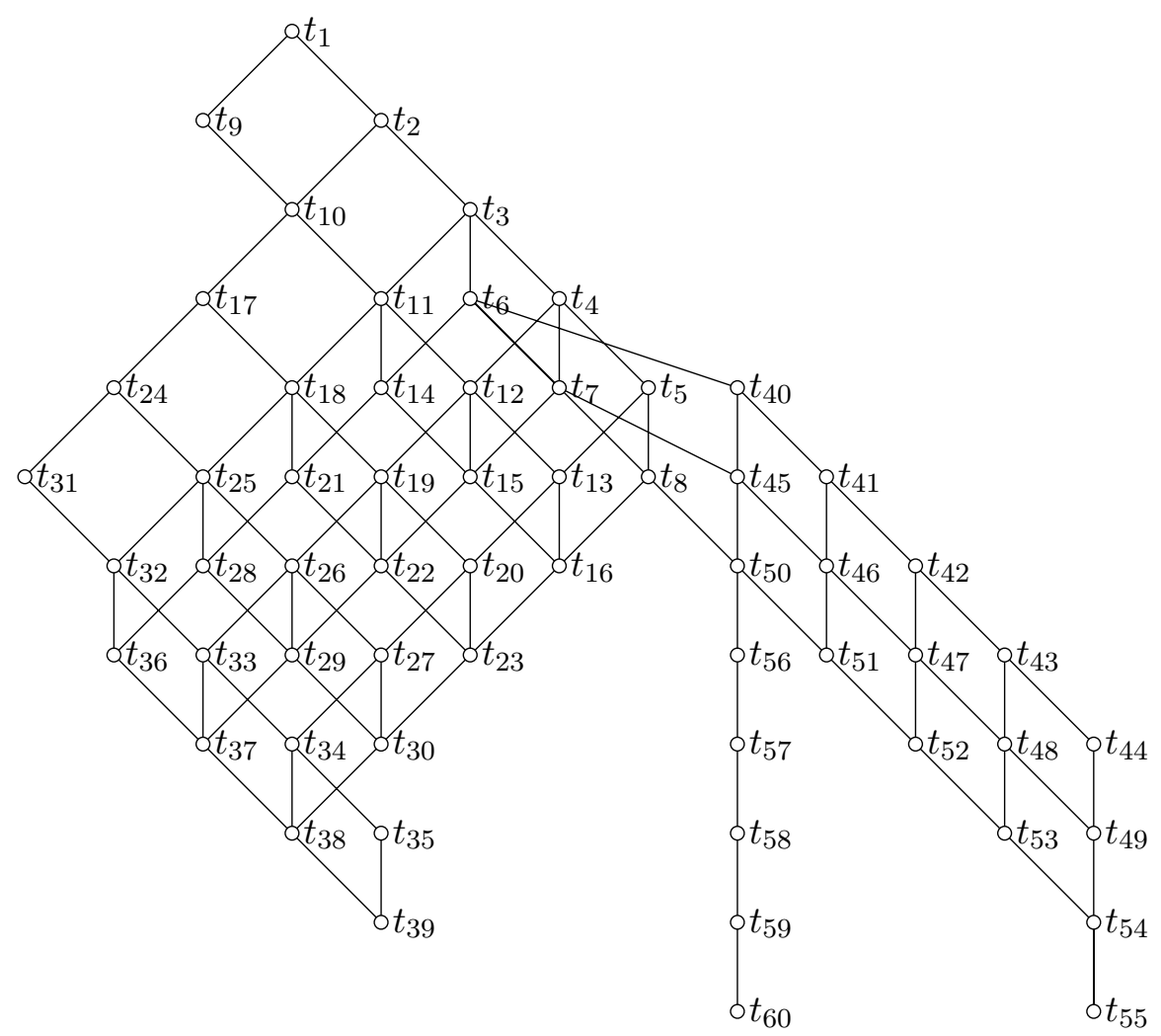

Fig. 9

except variables and products of two variables, can be obtained from the listed ones by renaming variables.

\section{Open Problems}

Problem 9.1. Is it true that every subvariety of $\mathcal{P}$ is generated by its order algebras?

Problem 9.2. Is every finite order algebra finitely based?

Problem 9.3. Is the variety $\mathcal{P}$ inherently nonfinitely based?

Problem 9.4. Are there any simple algebras in $\mathcal{P}$ that are not order algebras?

Problem 9.5. Characterize varieties of ordered sets. By a variety of ordered sets we mean an intersection of the class of all ordered sets (or order algebras) with a variety of groupoids. 
Based on the previous results, we are able to give the following examples of varieties of ordered sets. First of all, the class of all ordered sets, the trivial class of one-element ordered sets, the class of chains and the class of antichains are varieties of ordered sets. According to 3.3, the class of downrooted forests, i.e., ordered sets $\mathbf{A}$ such that $\{x \in A: x \leq a\}$ is a chain for any $a \in A$, is a variety of ordered sets. One can easily prove (or see [4] for a more general result) that an ordered set belongs to $\mathcal{P}_{\wedge}$ if and only if it is an ordinal sum of antichains. So this class is a variety of ordered sets. Also, the intersection of the last two varieties is the variety, consisting of the ordered

sets that are an ordinal sum of a chain and an antichain, with the antichain sitting at the top.

\section{REFERENCES}

1. J. A. Gerhard, The lattice of equational classes of idempotent semigroups, J. Algebra 15 (1970), 195-224.

2. P. Goralčík and V. Koubek, There are too many subdirectly irreducible bands, Algebra Universalis 15 (1982), no. 2, 187-194.

3. J. Ježek, Three-variable equations of posets, to appear in Czechoslovak Mathematical Journal.

4. J. Ježek and T. Kepka, Selfdistributive groupoids. Part D1: Left distributive semigroups, to appear.

5. J. Ježek, P. Marković, M. Maróti, and R. McKenzie, The variety generated by tournaments, to appear in Acta Univ. Carolinae.

6. J. Ježek and R. McKenzie, The variety generated by equivalence algebras, to appear in Algebra Universalis.

7. J. Ježek, M. Maróti P. Marković, and R. McKenzie, The variety generated by tournaments, to appear in Acta Univ. Carolinae.

8. D. J. Kleitman and B. L. Rothschild, Asymptotic enumeration of partial orders on a finite set, Trans. Amer. Math. Soc. 205 (1975), 205-220.

9. Ralph McKenzie, George McNulty, and Walter Taylor, Algebras, lattices, varieties, Volume I, Wadsworth and Brooks/Cole, Monterey, California, 1987.

10. Vladimír Müller, Jaroslav Nešetřil, and Jan Pelant, Either tournaments or algebras?, Discrete Math. 11 (1975), 37-66.

11. J. Neggers, Partially ordered sets and groupoids, Kyungpook Math. J. 16 (1976), 7-20. (Math. Rev. 55\#2675)

University of HaWait, Honolulu, HI

Charles University, Praha, Czech Republic

University of Cape Town, Cape Town, South Africa

VAnderbilt University, NashVille, TN 